\title{
Phylogeny of snakes (Serpentes): combining morphological and molecular data in likelihood, Bayesian and parsimony analyses
}

\author{
Michael S. Y. Lee ${ }^{1 *}$, Andrew F. Hugall ${ }^{1}$, Robin Lawson ${ }^{2}$ \& John D. Scanlon ${ }^{3}$ \\ ${ }^{1}$ Natural Sciences Section, South Australian Museum, Adelaide, SA 5000, Australia and School of Earth and Environmental \\ Sciences, University of Adelaide, SA 5005, Australia. \\ ${ }^{2}$ Osher Foundation laboratory for Molecular Systematics, California Academy of Sciences, Golden Gate Park, San Francisco, \\ CA 94118-4599, USA. \\ 3 Riversleigh Fossils Centre, Outback at Isa, PO Box 1094 Mount Isa, Qld 4825, Australia \\ submitted September 2004 \\ accepted December 2006
}

\section{Contents}

Abstract 371

Introduction 372

Morphological and molecular data 373

Methods 375

Separate analyses $\quad 375$

Combined parsimony analyses 375

Combined Bayesian analyses $\quad 375$

Combined likelihood analyses 376

Results 378

Separate analyses 378

Combined analyses 380

Discussion 383

Weighting morphology and molecules in parsimony and likelihood 383

Snake phylogeny and taxonomy 387

Acknowledgements 388

References 388

\begin{abstract}
The phylogeny of living and fossil snakes is assessed using likelihood and parsimony approaches and a dataset combining 263 morphological characters with mitochondrial (2693 bp) and nuclear (1092 bp) gene sequences. The 'no common mechanism' (NCMr) and 'Markovian' (Mkv) models were employed for the morphological partition in likelihood analyses; likelihood scores in the NCMr model were more closely correlated with parsimony tree lengths. Both models accorded relatively less weight to the molecular data than did parsimony, with the effect being milder in the NCMr model. Partitioned branch and likelihood support values indicate that the mtDNA and nuclear gene partitions agree more closely with each other than with morphology. Despite differences between data partitions in phylogenetic signal, analytic models, and relative weighting, the parsimony and likelihood analyses all retrieved the following widely accepted groups: scolecophidians, alethinophidians, cylindrophiines, macrostomatans (sensu lato) and caenophidians. Anilius alone emerged as the most basal alethinophidian; the combined analyses resulted in a novel and stable position of uropeltines and cylindrophiines as the second-most basal clade of alethinophidians. The limbed marine pachyophiids, along with Dinilysia and Wonambi, were always basal to all living snakes. Other results stable in all combined analyses include: Xenopeltis and Loxocemus were sister taxa (fide morphology) but clustered with pythonines (fide molecules), and Ungaliophis clustered with a boine-erycine clade (fide molecules). Tropidophis remains enigmatic; it emerges as a basal alethinophidian in the parsimony analyses (fide molecules) but a derived form in the likelihood analyses (fide morphology), largely due to the different relative weighting accorded to data partitions.
\end{abstract}

Key words Serpentes, character weighting, maximum likelihood, morphology, partitioned branch support, partitioned likelihood support

*Corresponding author. Email: Michael.S.Lee@ adelaide.edu.au 


\section{Introduction}

Despite well over a century of work, the higher-level relationships of snakes remain partly unresolved. While studies from only a few decades ago (Underwood 1967; Dowling \& Duellman, 1978; McDowell, 1975, 1987) often suggested radically different phylogenies, more recent morphological analyses (e.g. Rieppel, 1988; Kluge, 1991; Cundall et al., 1993; Tchernov et al., 2000; Lee \& Scanlon, 2002) have tended to retrieve a similar broad outline of snake evolution, with disagreement focused on particular taxa or regions of the tree, suggesting progress towards a resolved, well-corroborated phylogeny. However, recent analyses of molecular sequences (Slowinski \& Lawson, 2002; Wilcox et al., 2002; Vidal \& Hedges, 2002, 2004; Lawson et al., 2004) have called into question several (morphologically) well-supported clades, thus reopening the problem and suggesting that any resolution will require integration of all available sources of data and a re-evaluation of both molecular and morphological characters.

All recent morphological studies (Rieppel, 1988; Kluge, 1991; Cundall et al., 1993; Tchernov et al., 2000; Lee \& Scanlon, 2002) have retrieved several well-corroborated clades within snakes (see Fig. 1). The scolecophidians (worm-like blindsnakes and threadsnakes) are monophyletic and the most basal living snakes, though relationships within Scolecophidia remain disputed. The remaining living snakes also form a clade, Alethinophidia. The most basal alethinophidian lineages are the anilioids (e.g. uropeltines, Cylindrophis, Anomochilus, all at least semi-fossorial), but the relationships and even the monophyly of anilioids are uncertain. The remaining alethinophidians form a monophyletic Macrostomata (sensu lato: Rieppel, 1988; Lee and Scanlon, 2002). Xenopeltids (sunbeam snakes: Xenopelits and Loxocemus) are the most basal macrostomatans, but again, it is uncertain whether they are sister taxa, or successive outgroups to the remaining mac- rostomatans ('core macrostomatans': Lee \& Scanlon, 2002). The most basal core macrostomatans are the often large, constricting booids (boas, pythons, erycines), but again, their relationships and monophyly are uncertain. The remaining snakes form a clade informally termed 'advanced snakes' (Kluge, 1991). The most basal advanced snakes are the 'dwarf-boa' taxa: ungaliophiines, tropidophiines and bolyeriines. The remaining advanced snakes, acrochordids (filesnakes) and colubroids (colubrids, elapids, viperids) form a diverse and highly successful clade, the Caenophidia. This morphological phylogeny of living snakes supports the traditional view of snake evolution being characterised by progressive elaboration of the feeding apparatus and gradual loss of burrowing habits (e.g. Walls, 1940; Bellairs \& Underwood, 1951; Underwood, 1967; Cundall \& Greene, 1982, 2000; Greene, 1983, 1997; Rieppel 1988): scolecophidians, anilioids, xenopeltids and core macrostomatans have successively greater relative gape and more surface-active lifestyles. However, some palaeontological studies have challenged this scenario. There have been suggestions that some fossil snakes with relatively large gapes (the limbed marine pachyophiids and/or the large terrestrial madtsoiids) are basal (stem) snakes, and furthermore that the nearest well-known relatives of snakes are the macropredatory mosasaurs (e.g. McDowell, 1987; Scanlon, 1996; Caldwell, 1999; Lee, 1998, 2005a; Lee \& Caldwell, 2000; Lee \& Scanlon, 2002; Rage \& Escuillié, 2002, 2003). These phylogenetic patterns imply an alternative evolutionary scenario wherein some degree of enlarged gape was primitive for snakes, with gape either (1) being reduced convergently in scolecophidians and anilioids in response to feeding within the confines of narrow burrows (as has happened repeatedly within colubroids: Savitzky, 1983), or (2) reduced at the base of modern snakes and subsequently re-elaborated in extant macrostomatans. However, others (e.g. Zaher \& Rieppel, 1999; Tchernov et al., 2000; Rieppel et al., 2003) have suggested that

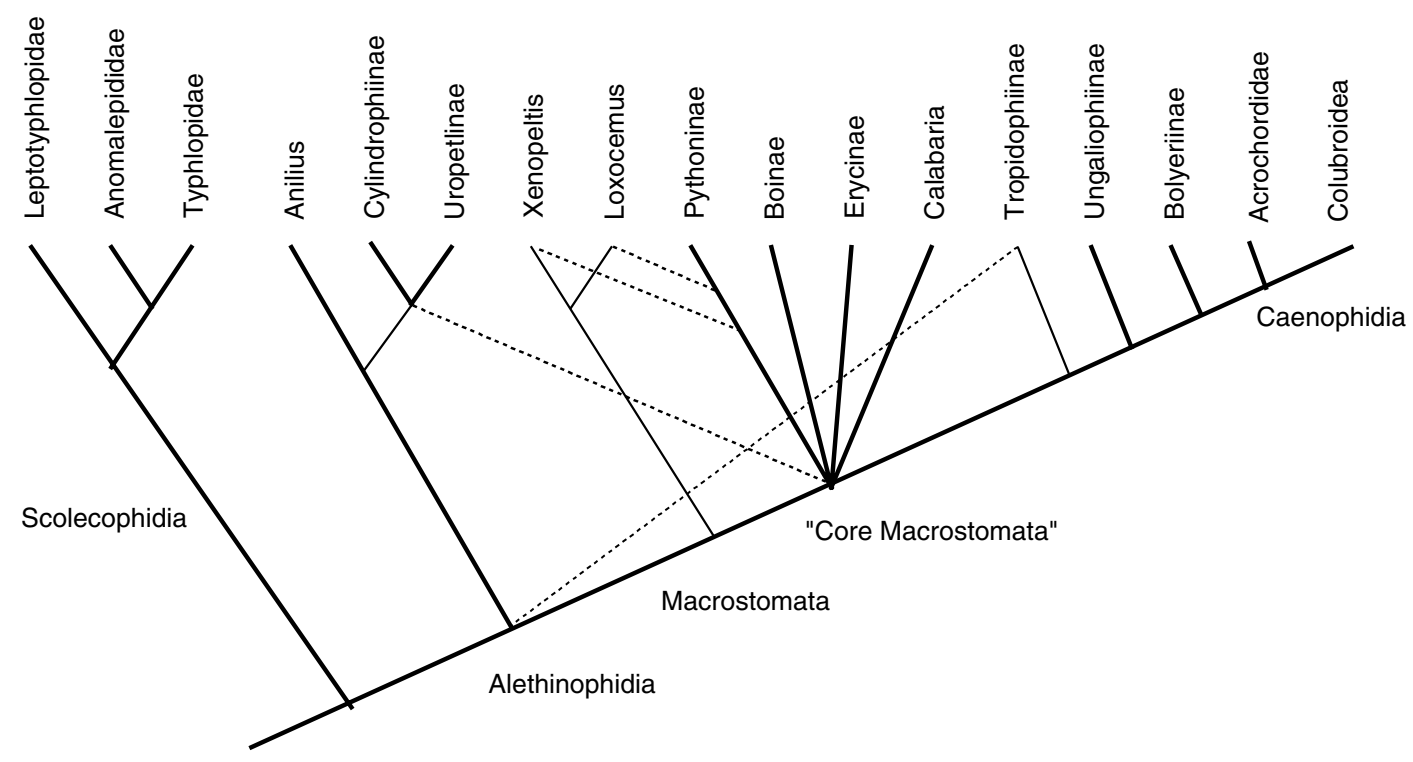

Figure 1 Currently accepted hypotheses of snake relationships, based on studies mentioned in the text. Thick lines denote relationships supported by both morphological and molecular studies (or supported by one and not strongly contradicted by the other). Thin lines denote relationships supported by morphological analyses, dotted lines denote relationships supported by molecular analyses. 
both pachyophiids and madtsoiids are true macrostomatans, and that the nearest outgroups to snakes are small fossorial squamates such as amphisbaenians and dibamids; these proposed relationships would be consistent with the traditional 'progressive' view of snake evolution. Regardless of the affinities of the disputed fossil snakes and their implications for evolutionary trends in certain character complexes (see overview in Lee \& Scanlon, 2002), all recent morphological studies have agreed in retrieving several major clades within living snakes (Scolecophidia, Alethinophidia, Macrostomata, 'core Macrostomata', 'advanced snakes', Caenophidia: Fig. 1).

Molecular studies have recently made important new contributions to snake phylogeny. Heise et al. (1995) used partial mitochondrial 12S and 16S rRNAs; Lawson et al. (2004) used complete mitochondrial cytochrome b; Slowinski and Lawson (2002) also used complete cytochrome b and included a sizeable ( $\sim 570$ bp) segment of nuclear c-mos; Wilcox et al. (2002) used an $\sim 1800$ bp mitochondrial sequence spanning $12 \mathrm{~S}, 16 \mathrm{~S}$ and intervening tRNAs; Vidal and Hedges used short fragments of cytochrome b, $12 \mathrm{~S}$ and $16 \mathrm{~S}$ with $\sim 570 \mathrm{bp}$ of c-mos (2002), and later $\sim 520$ bp of RAG 1 and 375 bp of c-mos (2004). These studies agreed with previous morphological conclusions in a few important respects (Fig. 1): the basal position of the scolecophidians within living snakes, and the monophyly of alethinophidians, of caenophidians and of colubroids. However, other morphological conclusions were contradicted, among them the monophyly and content of Macrostomata, core Macrostomata, and advanced snakes. For example, not all anilioids were basal to the remaining alethinophidians. While Anilius (along with tropidophiines) emerged as very basal, $\mathrm{Cyl}$ indrophis and uropeltines often appeared nested among 'macrostomatans'. Similarly, xenopeltids were not basal to core macrostomatans, but nested within them as close relatives of pythonines. However, the relationships of the dwarf boa lineages were most surprising. Ungaliophiines and trophidophiines were both removed from near caenophidians (thus breaking up the 'advanced snake' clade): ungaliophiines grouped with a boine-erycine clade, while tropidophiines were positioned in a highly heterodox position near the base of alethinophidians. These molecular results are not only incongruent with the morphological tree, but suggest extensive homoplasy in the evolution of gape and burrowing in snakes (Vidal \& Hedges, 2002), by placing some non-fossorial snakes with highly advanced gape (tropidophiines) near the base of the snake tree, and dispersing some apparently primitive, gapelimited burrowing forms (cylindrophiines, uropeltines and xenopeltids) among more derived snakes. Such trophic and ecological variability at the base of snakes is inconsistent with the traditional 'progressive' view of snake evolution, but is consistent with the view that the large-gaped and non-fossorial pachyophiids and madtsoiids are basal snakes (see Lee 2005b).

In cases where there are significant disagreements between data sets, the validity of a combined analysis has been debated (e.g. Bull et al., 1993). However, there are compelling arguments for simultaneous (or total evidence) approaches (e.g. Kluge, 1989; Nixon \& Carpenter, 1996), and it is now possible to assess data conflict in the context of combined analyses, obviating the need for partitioned analyses (Baker $\&$ deSalle, 1997). Also, the widely used incongruence length difference (ILD) test for assessing significance of conflict has problems (e.g. Barker \& Lutzoni, 2002), while methods such as reciprocal Templeton tests of the best tree for each data set are overly conservative, as they do not consider the possibility the full set of plausible trees for the different data sets might still contain trees in common. The possibility of informative interaction between 'incongruent' data sets can only be assessed in the context of combined analyses (e.g. Barrett et al., 1991; Gatesy et al., 1999; Lee \& Hugall, 2003). Several different data sets (however partitioned) might each contain the same underlying phylogenetic signal; however, in some or even most of these data sets, this signal could be swamped by noise (e.g. morphological convergence, base composition bias, random oversampling of 'poor' characters). Separate analyses of these data sets would retrieve different trees, and tests of incongruence might yield significant results. If the data sets are nevertheless combined, however, the misleading noise (which may be uncorrelated in independent data sets) might not be amplified, but the underlying phylogenetic signal (which should be congruent across different data sets) should be amplified, leading to improvements in phylogenetic estimation. This is the rationale behind the concept of 'hidden support', a prediction that has been demonstrated in empirical datasets (e.g. Gatesy et al., 1999; Wahlberg et al., 2005). For these reasons, a phylogenetic analysis of snakes is carried out based on a combined data set including the most comprehensive morphological and molecular data sets available to date, modified and in some cases expanded slightly to allow combination.

Typically, such combined morphological and molecular analyses have used parsimony. However, likelihood-based models may well be more appropriate for DNA data (Felsenstein, 1981, 2004). Thus, in addition to standard parsimony methods we also employ model-based (Bayesian and likelihood) methods. Using a model-based combined morphological and molecular analysis means that the morphology component of the tree 'score' needs to be translated into a likelihood value. Two possibilities are the 'no common mechanism' model of Tuffley and Steel (1997), and stochastic branchlength-based Markovian models described by Lewis (2001). In 'translating' combined analyses of morphological and molecular data from parsimony into likelihood the relative weight (likelihood differences across alternative trees) of the data partitions can change dramatically, further complicating matters.

\section{Morphological and molecular data}

The morphological data set of Lee and Scanlon (2002) was employed, with the following change. Because recent molecular analyses (Vidal \& Hedges, 2002, Lawson et al., 2004) did not retrieve a clade consisting of Calabaria and typical erycines (Erycinae sensu lato; Kluge, 1993), this terminal taxon was split into two and recoded accordingly (Calabaria and 'Erycinae sensu stricto'). While boine and/or erycine (s.s.) monophyly was not retrieved in some of these molecular 
studies (Lawson et al., 2004; Vidal \& Hedges, 2004), it was also not adequately refuted (based on bootstrap values of the conflicting nodes), and remains supported by morphology (see Kluge, 1991, 1993) Complete sequence data were also only available for one boine and one erycine genus. For these reasons, these terminal taxa were not subdivided further for this study. The morphological data set was combined with the rDNA data set of Wilcox et al. (2002), the protein-coding (mitochondrial cytochrome $b$ and nuclear c-mos) data of Slowinski and Lawson (2002), and the RAG1 data of Vidal and Hedges (2004). Heise et al. (1995) and Vidal and Hedges (2002, 2004) sequenced shorter sequences from $12 \mathrm{~S}, 16 \mathrm{~S}$ and c-mos regions, and their data were thus not used unless data from the other two studies were unavailable for the same taxa (see below).

As noted above, the number and identity of extant terminal taxa in this study were constrained by the availability of molecular sequence data. For each of the terminal ('family') taxa used in the morphological analysis, when this study was performed there was at most one included genus with complete sequences for all four regions. The molecular codings for these 'families' are based on this 'completely sequenced' genus. In most cases, all rRNA (Wilcox et al., 2002) and protein coding (Slowinski \& Lawson, 2002) regions were sequenced in the same species. In other cases, the rRNA study and protein-coding study sequenced different species from the same genus, and sequences from these closely related species have been concatenated. In most of these latter cases, there was only one species sequenced for RNA and one for proteincoding genes, and the sequences from these congeners were combined. However, both Typhlops jamaicensis and T. ruber were sequenced for rRNA, and T. platycephalus ('bradycephalus') was sequenced for the protein coding genes. Because jamaicensis is more closely related to platycephalus (Thomas, 1989; see also Wallach, 1999), the rRNA sequences for $j a$ maicensis were combined with the protein-coding sequences for platycephalus for the Typhlops sequence data. Similarly, Tropidophis greenwayi, feicki, melanurus and pardalis were sequenced for rRNA, and T. haetianus was sequenced for the protein coding genes. No comprehensive phylogenetic analysis of Tropidophis has been published, but greenwayi most closely resembles haetianus in dorsal scale rows and lack of keeled scales (Van Wallach, pers. com., 2003), thus the Tropidophis data consist of rRNA sequences from greenwayi combined with the protein-coding sequences for haetianus.

All terminal taxa have complete or nearly complete morphological data, and (except for the fossils and Anomochilus) at least partial mtDNA and nuclear data. However, Calabaria lacks some regions of the $12 \mathrm{~S}$ and $16 \mathrm{~S}$ data, since it was not sequenced by Wilcox et al. (2002), and shorter regions were used from the study of Heise et al. (1995); complete cyt b and c-mos fragment were generated recently (Lawson et al., 2004). Cylindrophis lacks RAG, as this taxon was not sampled by Vidal and Hedges (2004). Liotyphlops (Anomalepididae) lacks the 12S, $16 \mathrm{~S}$, and RAG sequences, but has at least some mtDNA and nuclear sequences. The outgroup sequence is a composite of two anguimorphs: $12 \mathrm{~S}$ and $16 \mathrm{~S}$ (Reeder unpubl. data), c-mos (Genbank AF435017) and RAG (Vidal and Hedges, 2004) all from Varanus and complete cyt b from Anguis (Slowinski \&
Lawson, 2002). Morphological (e.g. Lee \& Caldwell, 2000), molecular (e.g. Townsend et al., 2004) and combined (Lee, 2005b) studies have suggested that anguimorphs are close outgroups to snakes, though the molecular studies also suggested that iguanians might be equally close outgroups.

In using published sequence data we depend on the original author's assignations. Our own inspection of individual sequences and gene datasets showed no obvious evidence for pseudogenes or taxonomic mistakes. Further, both the c-mos and rag1 are single-copy genes in all complete genomes sequenced to date and phylogenies based on these genes (e.g. Townsend et al., 2004) show no evidence of paralogy in reptiles. As will be shown below, reasonable congruence beween the nuclear and mtDNA datasets suggests that the molecular data are clean, at least in regards to the phylogenetic questions relevant here.

The specimens examined for morphological data are listed in Lee and Scanlon (2002: Appendix 2), and the specimens sequenced are given in Wilcox et al. (2002: Section 2.1), Slowinski and Lawson (2002; Table 1), and Vidal and Hedges (2004: Appendix A). As these studies were performed by different researchers, different individuals (often of different species) were used when scoring each set of characters. In particular, the composite terminal taxa consist of morphological characters scored for clades of species, plus molecular characters sequenced for particular species in that clade. In combining the data into such composite terminal taxa, we rely on the original author's taxonomic assignations and current views about the phylogenetic position of the sequenced species (see details above). As an example, the morphological characters for Typhlopidae were based on 14 species in four genera, while the RNA sequences are based on Typhlops jamaicensis and the protein-coding sequences based on Typhlops platycephalus, neither of which were scored for morphology. Concatenating these observations into a composite taxon implicitly assumes that Typhlops jamaicensis and Typhlops platycephalus are more closely related to the typhlopid species scored for morphology than to any other species examined for morphological data. This assumption is currently well-supported (no recent workers have questioned the monophyly of Typhlopidae), but of course is subject to revision in the face of new data.

The alignments of Slowinski and Lawson (2002) and Wilcox et al. (2002) were preserved where possible; it was straightforward to add new sequences to these alignments using Clustal W (Thompson et al., 1997), adding extra gaps to the original alignments where necessary. Around $300 \mathrm{bp}$ of ambiguous alignment regions of the rRNA data were excluded from analysis (Wilcox et al., 2002), along with a short ( $\sim 62 \mathrm{bp}$ ) section of the $5^{\prime}$ end of the cytochrome b sequences. The number of characters in the three data partitions are: morphology, 263 (260 parsimony-informative), mitochondrial DNA, 2693 alignable (1093 parsimony-informative), nuclear DNA, 1092 alignable (170 parsimony-informative). The data matrix, with nexus commands showing excluded regions, is available from the Systematics and Biodiversity website and also at Cambridge Journals Online on: http://www.journals.cup.org/abstract_S1477200007002290. 


\section{Methods}

\section{Separate analyses}

Parsimony analyses were undertaken for the morphological, mitochondrial and nuclear data partitions, using PAUP* (Swofford, 2002). All trees were rooted with the anguimorph (lizard) outgroup. All searches (including bootstraps) were heuristic, employing 100 random-addition replicates. All multistate characters were treated as unordered; however, ordering certain morphological characters (when they corresponded to morphoclines) resulted in very similar trees (see Lee \& Scanlon, 2002). Support was determined using branch support (Bremer, 1988) and 1000 nonparametric bootstrap replicates.

Likelihood and Bayesian MCMC analyses were also undertaken for the mitochondrial and nuclear partitions, using PAUP (Swofford, 2002) and MrBayes 3.0b4 (Ronquist \& Huelsenbeck, 2003) respectively. Taxa totally lacking data for a data partition were deleted from the relevant analyses. In order to match the results to the analyses below (see Combined likelihood analyses), the HKYg model was used for both data partitions. PAUP parsimony analyses used heuristic searches with 100 random addition replicates, and 1000 nonparametric bootstraps to assess clade support; likelihood analyses used 100 random addition replicates for initial tree search, and 200 bootstraps with 'as-is' addition. The Bayesian MCMC analyses used 5 million steps $\times 4$ chains (heating $\mathrm{T}=0.2$, i.e. standard MrBayes settings), with sampling every 50 steps and a burn-in of 10000 samples (i.e. the first tenth), therefore posterior consensus trees were constructed from 90000 samples.

\section{Combined parsimony analyses}

The morphological, mitochondrial and nuclear data sets were concatenated and the combined data analysed using the search and rooting procedures described above. Branch support (Bremer, 1988) and partitioned branch support (PBS: Baker \& deSalle, 1997) were calculated using PAUP* commands generated by TreeRot (Sorenson, 1999) and modified where appropriate. PBS is the amount of support a particular data partition contributes to a clade in the context of a combined analysis, and can either be positive (improves support for the node) or negative (reduces support for the node). A PBS analysis with the full data set was first conducted. However, there are problems calculating PBS if certain taxa are completely lacking data for many partitions (the example discussed below involves fossil taxa lacking all gene sequences, but can also apply to poorly known extant taxa). When calculating PBS, the optimal tree containing clade $\mathrm{X}$, and the best constrained tree lacking clade $\mathrm{X}$, might only differ because the different position of fossil forms in the latter tree breaks up the clade. The PBS values for all genes for clade $\mathrm{X}$ will be zero since the two trees have identical backbone topologies for extant taxa. If the positions of the fossil taxa are particularly labile, this phenomenon can affect many clades, leading to many zero PBS values for every gene throughout the tree. When calculating PBS values for genes, a more informative approach would be to consider PBS values using only trees that differ in the relative position of taxa that contain sequence data. A simple but potentially problematic way to circumvent this problem would be to reduce taxon sampling to only include taxa with some sequence for each gene. However, this could severely reduce taxon sampling and seems unwise given the potential importance of dense taxon sampling for phylogenetic accuracy (e.g. Hillis, 1996). A more complicated but rigorous way to overcome this problem enables use of all available data in all tree calculations (Gatesy et al., 2003).

First, a combined analysis of the full matrix is undertaken ('full matrix tree'), and incomplete taxa are pruned, leaving a subtree where all taxa have data for all partitions (here called an 'extant subtree'). The PBS values for a clade on this extant subtree (clade X) can now be calculated. The relevant trees are the optimal tree (with an extant subtree containing clade $\mathrm{X}$ ), and the best tree with an extant subtree that lacks clade $\mathrm{X}$. The latter tree can be found using reverse backbone constraints in PAUP*. The complete data set is used for all analyses; in particular, the fossil taxa are included but left to 'float'; they are not omitted because their character states can still influence the interrelationships of extant taxa. To calculate PBS, data partition $\mathrm{A}$ is optimised onto the first tree (with all taxa included) and the tree length measured ( $\mathrm{L}_{\text {TreeWithSubtreeWithCladeX, } \mathrm{A}}$ ).

Data partition $\mathrm{A}$ is then optimised onto the second tree (with all taxa included), and the tree length measured $\left(\mathrm{L}_{\text {TreeWithSubtreeLackingCladeX, A }}\right)$. The length difference is the PBS for Clade $\mathrm{X}$ for data partition $\mathrm{A}$ :

$$
\begin{aligned}
\text { PBS }_{\text {CladeX, A }=} & \mathrm{L}_{\text {TreeWithSubtreeLackingCladeX, A }} \\
& -\mathrm{L}_{\text {TreeWithSubtreeWithCladeX, A }}
\end{aligned}
$$

In the current analysis, PBS values were calculated using a backbone subtree containing taxa with at least some information for both morphology and molecules. Thus, five taxa which lacked molecular data (four fossil forms, and Anomochilus) were excluded. Their exclusion from the PBS analysis resulted in the elimination of the numerous zero values found in the initial PBS analysis which considered all taxa (Fig. 3 and 4). A PAUP* script to conduct this search is embedded in the Nexus data file.

Bootstrap values (1000 replicates) were also calculated using PAUP*. The values shown in the results are for the tree including all taxa, and for the backbone subtree of 'extant' taxa (i.e. with both morphological and molecular data). In the latter tree, the simple way of deleting incomplete taxa before conducting the bootstrap is potentially problematic since these taxa might influence the relative relationships of the complete taxa. The approach used here was to conduct a bootstrap using the complete data set, save all the trees, and then prune the incomplete taxa from these trees before compiling a majorityrule consensus of these pruned trees.

\section{Combined Bayesian analyses}

A Bayesian MCMC run was performed using the same models as the Mkv + HKYg analyses above, with the morphology employing the standard stochastic model (Mkv-type) in MrBayes, and the nuclear and mitochondrial partitions assigned separate 
HKYg models (see above). Because autapomorphies were excluded from the morphological data (as is normal procedure), terminal branch lengths are going to be artificially shortened for this data set (e.g. Bromham et al., 2002). The molecular data sets, in contrast, exhibit no such bias. Thus, unlike the morphological branch lengths, the branch lengths for different molecular data sets could be expected to be similar to each other, all being correlated with time elapsed. For this reason, branch lengths were unlinked between the morphological and molecular data, but linked between the two molecular data sets. Four (one cold, three heated) 5 million step MCMC chains were run, sampling every 50 generations, with the first 5000 samples discarded as burn-in, leaving 95000 trees for construction of a majority rule consensus.

\section{Combined likelihood analyses}

The morphological data were analysed as unordered (see discussion above) using two different likelihood models that approximate parsimony, the 'no common mechanism' model (NCMr; Tuffley \& Steel, 1997) and the stochastic 'Markov' model (Mkv; Lewis, 2001). Calculation of the NCMr model score is simple (see equation 42 Tuffley \& Steel, 1997):

$$
-\ln \mathrm{L}=\sum_{i=1}^{k}\left(l_{i}+1\right) \ln \left(r_{i}\right)
$$

where $l_{i}=$ parsimony length of a character, $r_{i}$ the number of states in that character, for $k$ characters. Effectively each character has its own 'optimal' branch lengths, equal to the parsimony steps for that character (for this reason, invariant characters do not affect branch length estimation with this model). The ' $r$ ' in NCMr refers to the number of character states, which is free to vary for different characters. As can be seen from the above formula, when $r$ is the same for all characters the $\mathrm{NCMr}$ model is equivalent to MP. However, when $r$ varies, the $\mathrm{NCMr}$ model deviates slightly in assigning different weights to characters with different numbers of character states: likelihood among equally parsimonious trees can therefore vary, and the relative ranking of trees can differ. The 263 morphology characters include 172 where $r=2,82$ where $r=3$ and 9 where $r=4$; here the NCMr model is not exactly equivalent to MP.

For Markovian models (Mkv where k and v represent, respectively, the number of character states and adjustment for presumed missing invariant sites; see below), branch length estimates are essential to the calculation of likelihood and thus estimation of ML topologies. Because morphological datasets typically exclude invariant (as well as autapomorphic) characters, branch length estimation will be compromised. Mkv models as outlined by Lewis (2001) use a conditional likelihood adjustment to account for invariant sites, based on the likelihood of dummy invariant characters (Felsenstein, 1992). Markovian models do not match parsimony but can be made to converge with MP by the addition of more and more invariant sites (Tuffley \& Steel, 1997; Steel \& Penny, 2000), a situation analogous to the conditional likelihood adjustment.

There is presently no program for calculating the likelihood of a topology for the Mkv model with full branch length optimisation, where k differs among sites. Therefore, we employed a two-stage approximation using PAUP* to calculate the morphological data Mkv model $\operatorname{lnL}$ for any given topology. First, all the morphological characters are recoded as DNA states and 60 dummy invariant sites added (inserted manually into the nexus file). With these data, the Jukes and Cantor (1969) model then provides branch lengths for a given topology. This is a Mkv model where $\mathrm{k}=4$ for all characters and is therefore only a (in this case reasonable) approximation of the Mkv model maximum likelihood estimation of branch lengths. Sixty dummy characters were used as it represented the minimum required for likelihood to recover the same topology as parsimony (for the morphological data) but did not attribute excessive weight to the morphological data in combined analyses (see below). This tree (now with its branch lengths fixed) is then used to calculate likelihoods for 2-state, 3-state and 4state character sets separately. This was done by manipulating the base content parameter to allow only 2, 3 or 4 states. The scores for the 2, 3 and 4 state character sets were then added to give the final total $\operatorname{lnL}$ for that given topology.

The Tuffley and Steel model (NCMr, where $r$ represents the number of character states) has been criticised as too complex with many incidental parameters and therefore potentially statistically inconsistent and biologically unrealistic (Steel \& Penny, 2000; Lewis, 2001). Conversely, the assumptions of the Mkv model might suit morphological datasets better if an attempt was made to score all characters; however, these datasets were usually intended for parsimony analysis and thus purged of autapomorphic as well as invariant characters (see Yeates, 1992). However, such concerns are not strictly relevant given the aims of this paper, which is to use likelihood for the DNA data but to incorporate an analysis of the morphology that mimics 'standard' parsimony as closely as possible. Accordingly, we have assessed how closely these likelihood morphological models approximate parsimony. For 10000 random trees, the NCMr likelihoods showed a much tighter correlation with parsimony scores (Spearman rank correlation coefficient $=0.997)$ than did the Mkv likelihoods $(\mathrm{cc}=0.717$; see Fig. 2). Increasing the number of dummy sites tenfold (to $600)$ improved the correlation of the Mkv model $(\mathrm{cc}=0.924)$ but it still did not approach the NCMr model (Fig. 2); moreover, this procedure accorded very high (arguably excessive) weight to the morphological data, relative to the molecular data.

Hierarchical likelihood ratio tests suggested that the best fitting model for each gene were as follows: rRNA, GTRig; Cytb, TVM + ig; c-mos, HKYg; RAG1, TrNg. However, these tests can be overly sensitive given finite data (e.g. Felsenstein, 2004); furthermore, oversplitting the data and using complex models increases the risk that the partitions are too small for accurate parameter estimation. A complex analysis (in MrBayes) where each gene was assigned its selected model yielded very similar topologies and supports to a simpler analysis where two partitions (mitochondrial and nuclear DNA) were assigned separate HKYg models (a special case of every selected model; Hasegawa et al., 1985; Yang, 1994). This suggests that the simpler models did not provide misleading results. Therefore, for the sake of practicality in an already complex task, simpler models were used in the analyses below, as it permitted more 


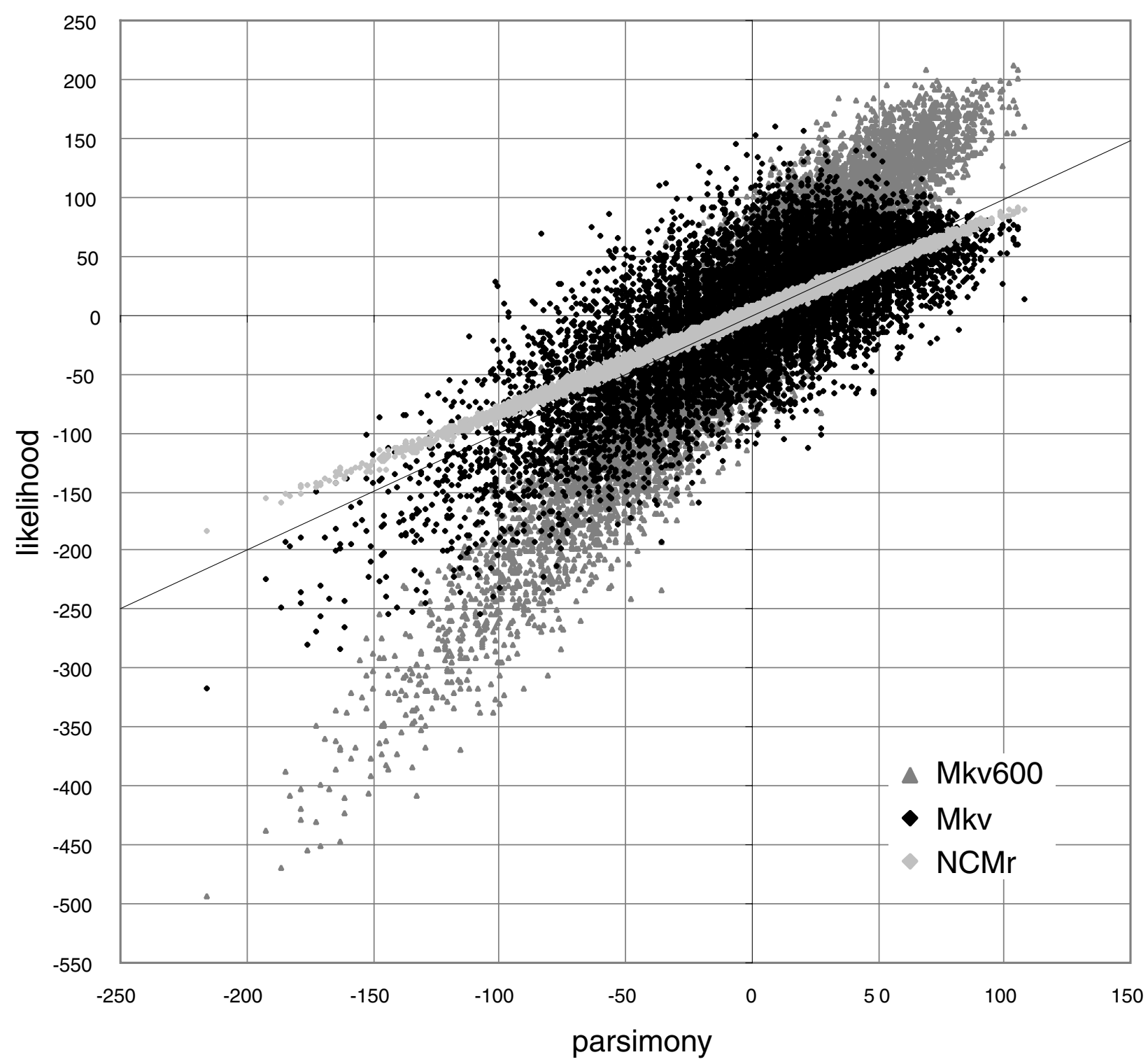

Figure 2 Correlation of the NCMr and Mkv likelihoods (calculated as described in methods) with parsimony scores (tree length) for the morphological data set, based on 10000 random trees generated by MacClade. Mkv60o is Mkv model using a large number of dummy invariant sites (600 compared with 60 for Mkv), giving a tighter correlation with MP but a much higher weighting (see results). The relative slope can be seen as an indication of the 'weighting'; Black line indicates 1:1 slope $=$ MP.

rapid evaluation of tree likelihoods and branch supports using PAUP*.

Exact tree searches implementing the likelihood morphology models above with standard DNA models could not be performed using any available phylogenetic analysis programs and had to be conducted manually, using a procedure that mirrored heuristic searches. This first involved gathering a large number of potentially optimal ('candidate') topologies. All the optimal and near-optimal trees (for the combined data) found in the parsimony analyses were pooled, along with all the trees sampled in the Bayesian MCMC analysis (see above). Reverse-constraint parsimony searches for every node on the best parsimony tree and the MCMC consensus tree were also undertaken, and the optimal and near-optimal constrained trees added to the tree pool. For reasons discussed under 'Parsimony', the reverse constraints analyses were performed for nodes in the backbone subtree of 18 taxa, but all taxa were included in tree searches and tree scores. The diversity of starting trees (62 035 candidate topologies generated using several different methods) made it less likely the subsequent analysis became trapped in a local optimum.

For each of these starting candidate topologies, $\operatorname{lnL}$ scores for the morphology, mtDNA and nuclear DNA partitions were calculated. Both mtDNA and nuclear DNA partitions used the HKYg model (each with their own optimal parameter and branch length estimations). For the morphology, both $\mathrm{NCMr}$ and Mkv $\operatorname{lnL}$ were calculated. Then by summing the scores of the partitions we get a total $\operatorname{lnL}$ score for each topology, for both 
combinations of models: morphology with NCMr plus DNA with $\mathrm{HKYg}$, and morphology with Mkv plus DNA with HKYg. In this procedure we have calculated the $\ln L$ for each partition using a model and branch lengths specific to that partition, and then combined the $\operatorname{lnL}$ scores. This method of combining data is analogous to analyses in MrBayes where branch lengths are unlinked (see Jamieson et al., 2002; Seo et al., 2005).

The best tree in the set of candidate topologies for each model combination is then identified, along with the best reverse constraint trees for each node in the best tree. MicroSoft Excel and JMP (SAS Institute Inc.) were used to collate scores. These best, and best reverse-constraint, trees from the two analyses were then subjected to branch-swapping using PAUP* (settings: NNI and TBR with rearrangement limit=3). All new unique topologies generated were added to the pool of candidate trees and assessed, with any new best tree identified. Both NCMr and Mkv analyses were conducted together, the trees generated from each model together contributing to the growing common pool of candidate topologies. If there was a new best tree from either analysis, the best reverse constraint trees were again identified, and this set of trees again subjected to the branch swapping procedure, as described above. After four rounds, no new best trees were found for either analysis $(\mathrm{NCMr}+\mathrm{HKYg}, \mathrm{Mkv}+\mathrm{HKYg})$. In total 110899 topologies were tested.

A measure of clade support was determined from these trees by constructing a majority-rule consensus with each tree weighted in proportion to its likelihood (see Jermiin et al., 1997; Strimmer \& Rambaut, 2002); thus, trees with higher likelihood contribute exponentially more strongly to this consensus. Only the best 10000 trees were included in this consensus as the weighting of the remainder becomes trivial; there is negligible difference between using 5000 best and 20000 best. Of course, many trees (in particular, many very poor trees) were not considered in this consensus, but their likelihoods are so minute that they would not be expected to change the consensus values. Analogously, many very poor trees will be unsampled in a finite number of bootstrap replicates or MCMC chains but their non-zero probabilities (given infinite sampling) are so small that this omission has little effect on bootstrap or MCMC support measures.

Partitioned likelihood support (PLS: Lee \& Hugall, 2003) was used to evaluate the agreement and relative signal strength of the three data sets in combined likelihood analyses. The rationale and methodology for calculating PLS is similar to that for PBS. Again, an attempt was made to use the entire matrix rather than to prune it down to only 'extant' taxa. The full morphological and molecular data set was analysed using likelihood (as above) and the optimal tree obtained. Based on the arguments presented under Parsimony Analyses, PLS values were calculated only for clades in a subtree containing the 18 taxa with data for both morphology and molecules. However, as before the full 23-taxon data set was used in tree searches and in calculation of tree scores when assessing support for nodes in the 18-taxon subtree.

For a given clade (clade $\mathrm{X}$ ) on this subtree, the partitioned likelihood for a data partition (partition A) is calculated in exactly the same fashion as for PBS (see above), except that likeli- hood methods were used in tree searches and data optimisation. Briefly, data set A is optimised on the ML tree for the full data set (which contains this subtree with clade $\mathrm{X}$ ), and the negative $\log$-likelihood calculated ( $-\ln L_{\text {TreeWithSubtreeWithCladeX, A) }}$. Then, this data set is optimised on the constrained tree, i.e. the best tree found for the full data set which has a subtree lacking clade $\mathrm{X}$, and the negative log-likelihood calculated $\left(-\ln L_{\text {TreeWithSubtreeLackingCladeX, A }}\right)$. The difference between these values is the PLS for data set A:

$$
\begin{aligned}
\text { PLS }_{\text {CladeX, A }}= & -\left(\ln L_{\text {TreeWithSubtreeLackingCladeX, A }}\right) \\
& -\left(-\ln L_{\text {TreeWithSubtreeWithCladeX, A }}\right) \\
= & \left(\ln L_{\text {TreeWithSubtreeWithCladeX, A }}\right) \\
& -\left(\ln L_{\text {TreeWithSubtreeLackingCladeX, A }}\right)
\end{aligned}
$$

As with PBS calculations, all taxa and characters were used in the analyses, but the five taxa missing molecular data were allowed to float. However, because there is no program that can do standard and reverse ML searches with morphological and molecular data, only manual heuristic searches could be employed (see above).

\section{Results}

\section{Separate analyses}

The morphology tree is shown in Fig. 3a, and is identical to that obtained from a previous study (Lee \& Scanlon, 2002). The mtDNA trees obtained from the likelihood and Bayesian analyses were very similar to one another, and in broad agreement with previous mtDNA results (Slowinski \& Lawson, 2002; Wilcox et al., 2002). Bootstrap frequencies were generally lower than Bayesian probabilities, but were generally well correlated nonetheless (Fig. 3b). The parsimony mtDNA tree (strict consensus of 4 trees) was slightly different but most conflicts affected nodes poorly supported in the model-based trees (see clades marked ' $x$ ' in Fig. 3b). The nuclear DNA trees from the likelihood and Bayesian analyses are very similar to one another (Fig. 3c) and to those from previous nuclear analyses (Slowinski \& Lawson, 2002; Vidal \& David, 2004). Again, bootstrap frequencies were generally lower than, but correlated with, Bayesian posteriors. The parsimony nuclear tree (strict consensus of 9 trees) was poorly resolved, probably as a consequence of fewer variable sites coupled with short branches in basal alethinophidians (as suggested by the mtDNA analyses); however, the few strongly resolved clades were all congruent with likelihood and Bayesian trees.

We then obtained strict and semistrict (combinable component) consensus trees of the morphology tree and the ML trees for the mt and nuclear DNA (Fig. 3a-c); both approaches yielded the same, poorly resolved tree (Fig. 3d). The fossil taxa and Anomochilus, being present only in the morphological tree, were excluded during the construction of this consensus, and then re-inserted into this consensus tree based on their morphological positions. However, these consensus methods are conservative in that they retrieve only clades uncontradicted by any dataset; majority-rule and Adams consensus trees could be better resolved. 
A
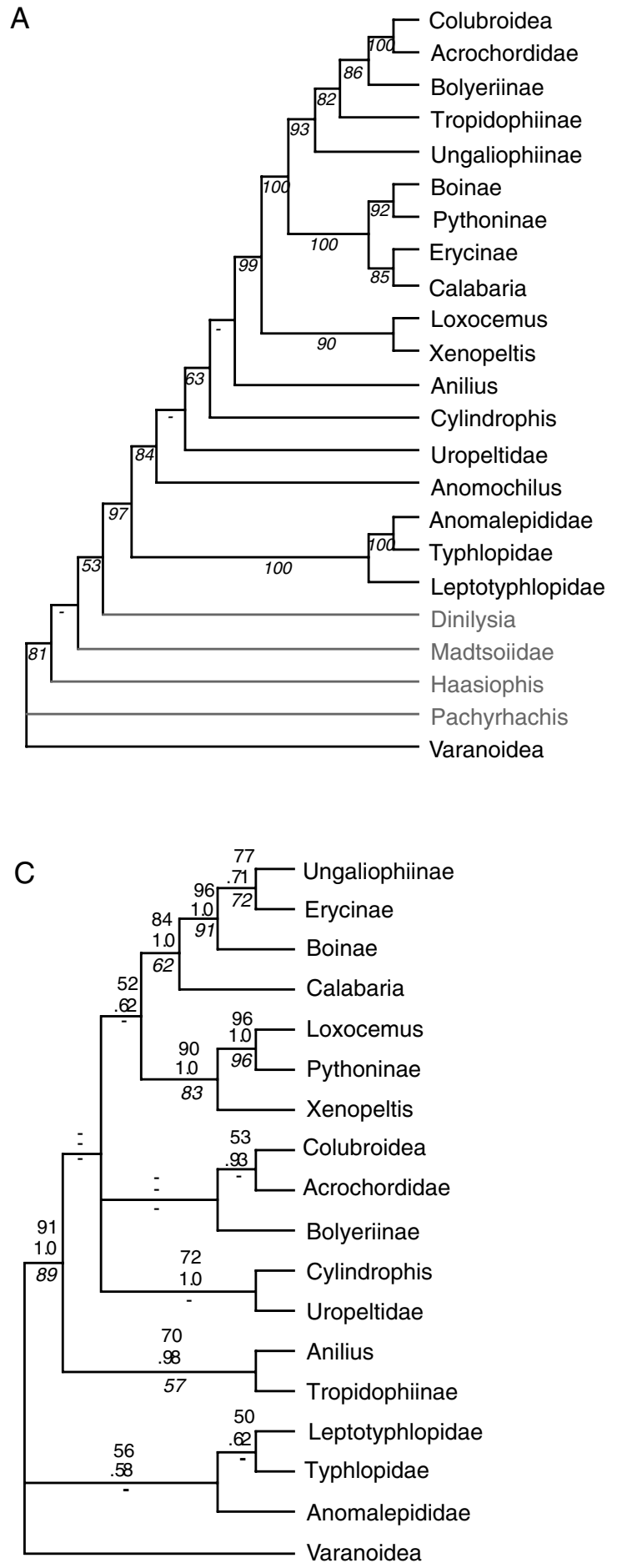

B
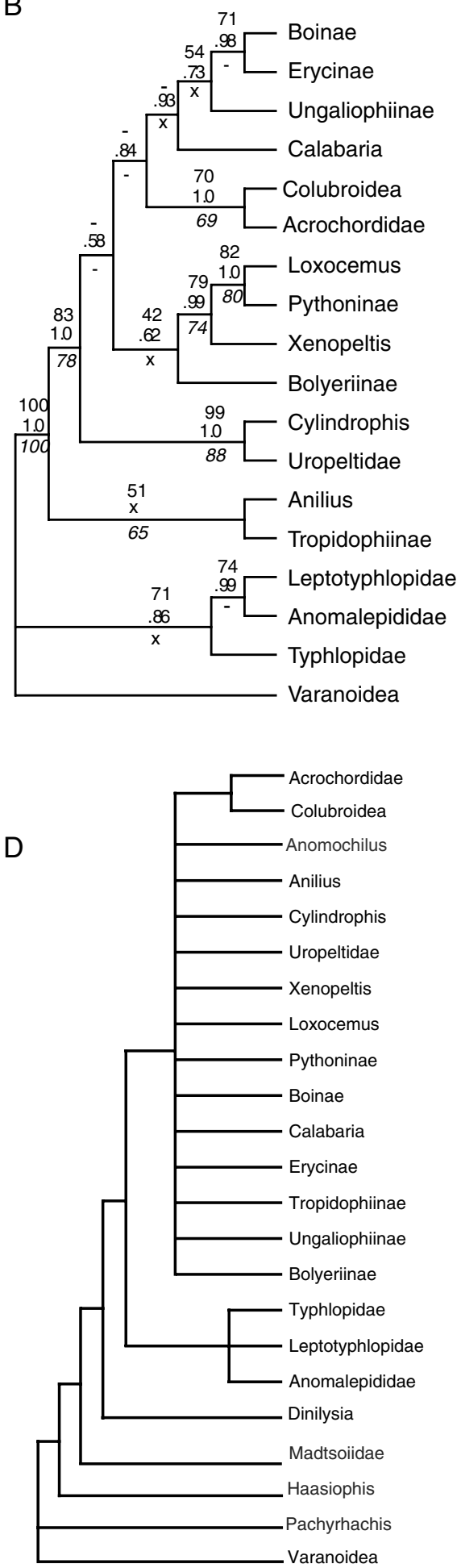

Figure 3 Trees for the various separate analyses. Fossil taxa in grey, living taxa in black. (a) Morphology, based on parsimony analysis. Length $=730$. Numbers below branches in italics are bootstrap frequencies. (b) mtDNA, based on maximum-likelihood analysis using the HKYg model. $-\ln =22691.42$. Numbers above branches refer to likelihood bootstraps, and Bayesian posteriors respectively, numbers below branches (in italics) refer to parsimony bootstrap frequencies. "-" indicates that the clade is either present or consistent with the results of the parsimony or Bayesian analysis (best tree or strict consensus), but has a bootstrap or posterior of less than $50 \%$; " $x$ " indicates the clade is inconsistent with the results of the parsimony or Bayesian analysis. (c) nuclear DNA, based on maximum-likelihood analysis using the HKYg model; this best tree constrains a polytomy. $-\ln =4859.34$. Numbers on branches refer to, respectively, likelihood bootstraps, Bayesian posteriors, and parsimony bootstraps. "-" and " $x$ " as for fig. b.

(d) The strict and semistrict consensus of trees a-c. Positions of fossil taxa and Anomochilus are based on morphology only. 


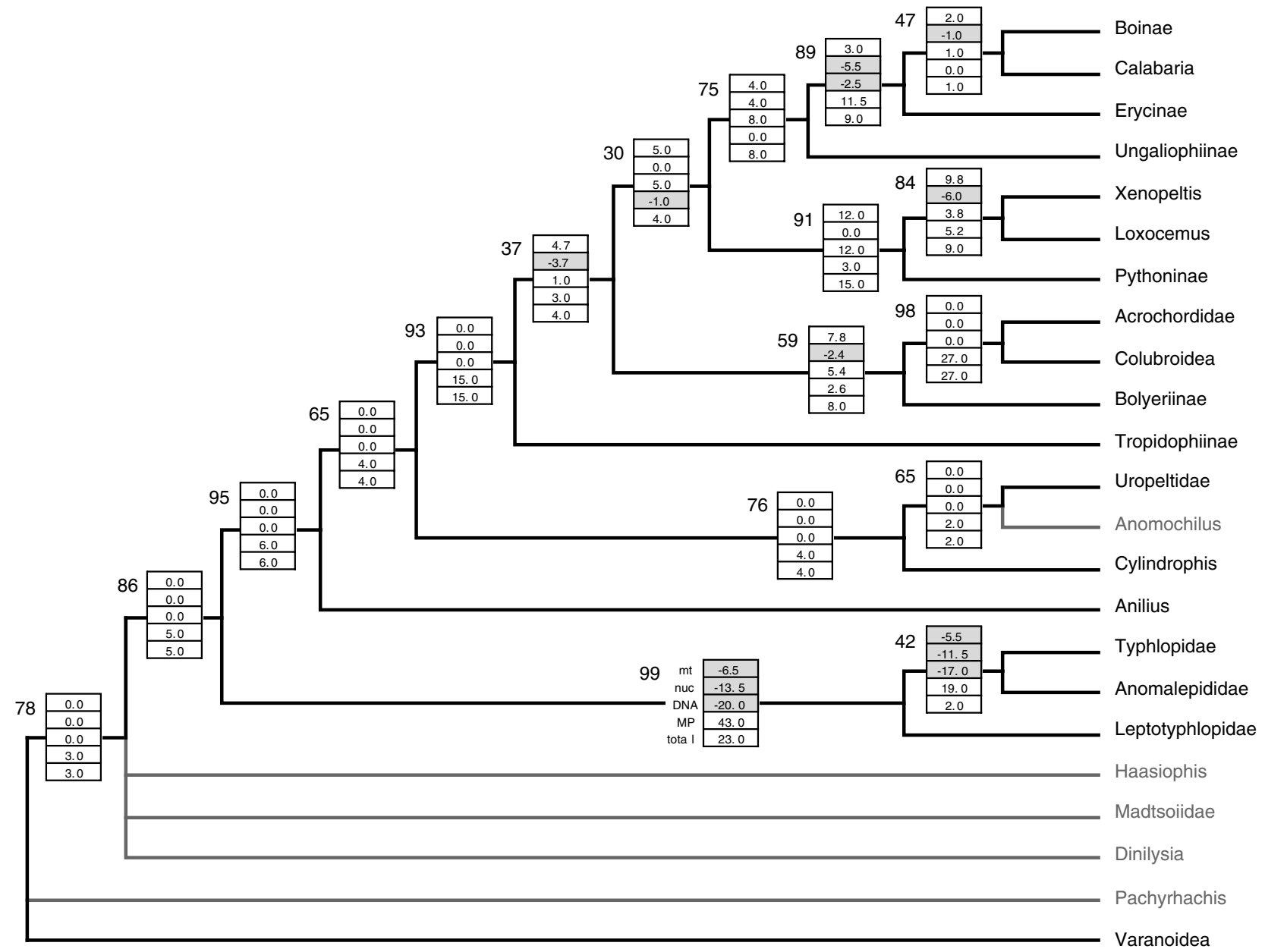

Figure 4 Strict consensus of the two MP trees $(\mathrm{L}=6640)$ for snakes, based on combined analyses of morphological, mitochondrial, and nuclear data partitions. Bootstrap and Partitioned Branch Support values for each clade are indicated. The five morphology-only taxa are in grey. Note the numerous zero PBS values caused by five taxa entirely lacking the two molecular data partitions, a problem addressed in the pruned tree (Fig. 4). The five values in the PBS boxes are from top to bottom, mtDNA, nuclear, sum DNA, morphology, sum total.

\section{Combined analyses}

The parsimony, Bayesian and ML trees were similar in many respects. As a result, the strict consensus of the two most parsimonious trees will be discussed in some detail, followed by discussion of where the Bayesian and ML trees differ. The optimal tree for all taxa found in the parsimony analysis, and bootstrap and PBS values, is shown in Fig. 4. The parsimony tree for the 18 'extant' taxa, with tree searches, bootstraps and PBS values performed using the full 23-taxon matrix (see methods), is shown in Fig. 5. The first tree contains many zero PBS values for genes; this is not a result of lack of molecular signal, but is an artefact created by the ambiguity in the position of the taxa lacking molecular data (see methods). Discussion of PBS and the relationships of extant taxa will thus focus on the second tree (Fig. 5). These parsimony trees contain elements suggested by recent analyses of either morphological or molecular data, or both. However, there is extensive conflict between the data sets: only two of the 15 clades have positive PBS for all three data partitions, while a further three have positive PBS for one partition that is not strongly contradicted by the other two partitions (PBS between 0 and -2). Pachyrhachis is the most basal snake, followed by the other three fossil taxa (Lee \& Scanlon, 2002). As suggested by all recent morphological and molecular analyses, scolecophidians (blindsnakes) are the most basal extant snakes. Scolecophidian monophyly is strongly supported by the morphological data, but contradicted by a single molecular study (Heise et al., 1995). Here, parsimony analyses of the molecular data alone (combined $\mathrm{mt}+$ nuc) also show scolecophidian paraphyly.

Monophyly of alethinophidians (all extant snakes excluding scolecophidians) is supported by all data partitions. Anilioids are paraphyletic, with Anilius basal to the remaining alethinophidians, consistent with some recent molecular studies (Wilcox et al., 2002; Lawson et al., 2004). In the complete tree (Fig. 4), the enigmatic Anomochilus falls in a Cylindrophis-uropeltine clade, sister to the remaining snakes. Relationships among remaining alethinophidians (macrostomatans sensu lato) include some traditional elements long recognised from morphological analyses along with new clades suggested by molecular analyses, especially the diphyly of the 


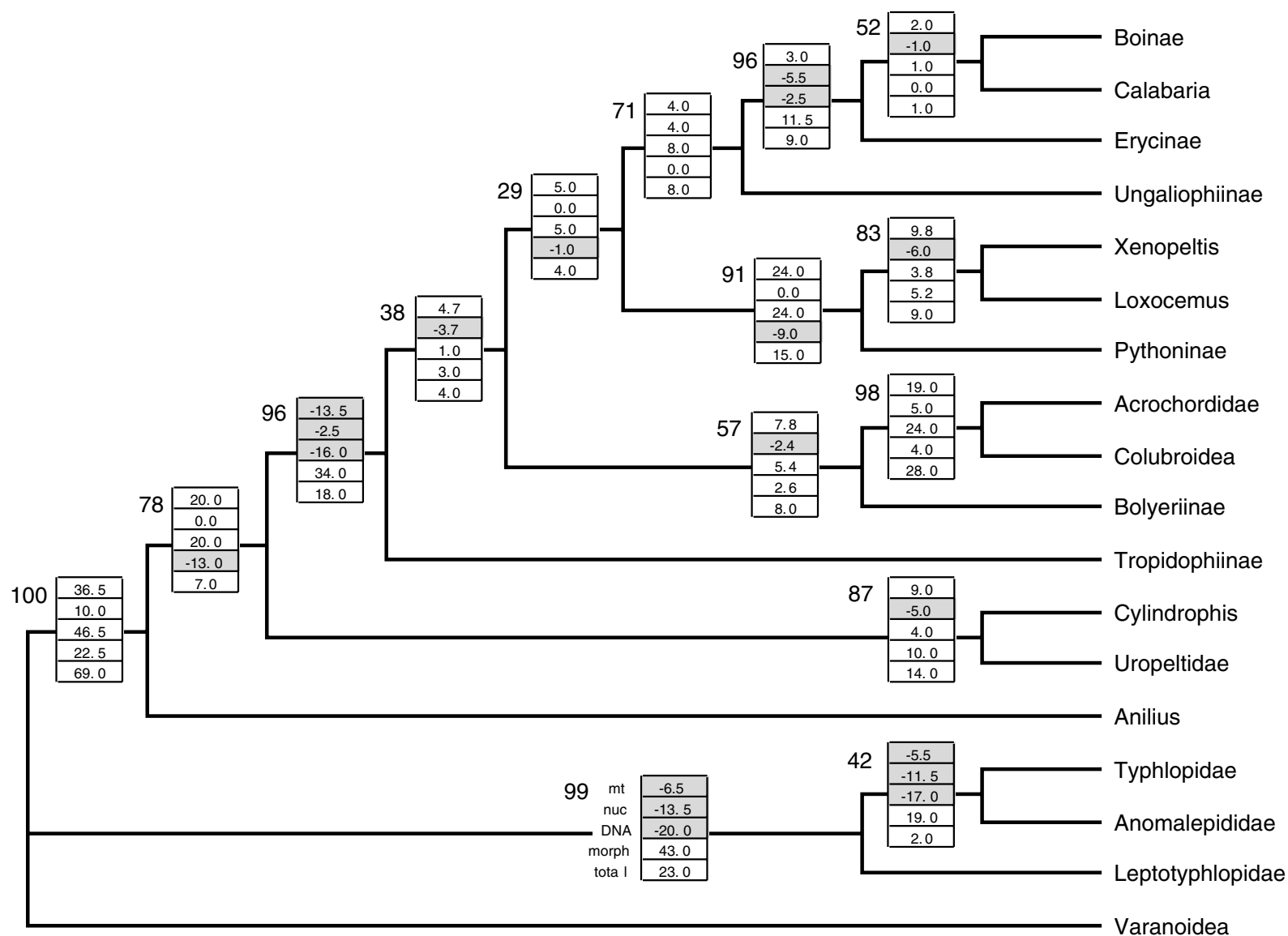

Figure 5 MP tree for the 18 'extant' snake taxa plus outgroup, based on combined analyses of morphological, mitochondrial, and nuclear data partitions for all 23 taxa and followed by pruning five taxa without DNA data (four are fossil forms). Bootstrap values for each clade are indicated; these were calculated using the full (23-taxon) data set, with the 5 taxa without DNA data pruned from the best tree(s) from each bootstrap. PBS values are also shown; these were again calculated using the full 23-taxon dataset, with each clade in this tree being used as a reverse backbone constraint (see text).

dwarf boas (tropidophiines and ungaliophiines). Recent morphological analyses had foreshadowed this result by removing ungaliophiines from a sister relationship with tropidophiines (Zaher, 1994; Lee \& Scanlon, 2002), but still placed one or both of them near the advanced snake clade (bolyeriines, acrochordids and colubroids). Tropidophiines here emerge as basal macrostomatans, approaching the unexpectedly basal position found for them in recent molecular analyses (Slowinski \& Lawson, 2002; Wilcox et al., 2002; Vidal \& Hedges, 2004) and contradicting the higher position suggested by morphological studies (e.g. Underwood, 1967; Cundall et al., 1993; Lee \& Scanlon, 2002). Ungaliophiines group with boids (erycines, boines and Calabaria), as suggested by molecular (Wilcox et al., 2002; Lawson et al., 2004) and some morphological (Zaher, 1994) analyses. The position of xenopeltines (Xenopeltis and Loxocemus) again represents a compromise between traditional morphological and recent molecular views. As suggested by morphological data (e.g. Underwood, 1967; Lee \& Scanlon, 2002), the two genera are sister taxa; however, they are not basal to other macrostomatans $s$. $l$., but are closely related to pythonines, as suggested by recent molecular analyses (Heise et al., 1995; Slowinski \& Lawson, 2002; Wilcox et al., 2002).
The monophyly of caenophidians (Acrochordus and colubroids), and their affinities with bolyeriines, is consistent with traditional views (e.g. Cundall et al., 1993; Lee \& Scanlon, 2002), and these clades are supported by morphological and at least one molecular data set. Similarly, the monophyly of boids (boines, Calabaria and erycines) has been proposed based on morphology (e.g. Cundall et al., 1993, but see Lee \& Scanlon, 2002), and is again supported by morphological and one molecular data set.

The ML analysis employing the Mkv morphology model and the HKYg molecular models (Mkv + HKYg) yielded a best tree (Fig. 6) with $-\ln \mathrm{L}=30464.989$ (morphological component $-\ln \mathrm{L}=2816.246$, DNA component $-\ln \mathrm{L}=27648.743$ ). This tree was almost identical to the parsimony tree. Likelihood weighted support for the full tree, and the subtree of reasonably complete taxa, is shown in Fig. 6. The only strongly supported difference was that tropidophiines appear higher up than in the parsimony tree (near caenophidians), more closely reflecting morphological views. Again, the PLS values for most clades showed some conflict; however, congruence improved with Scolecophidia (in addition to Alethinophidia and Caenophidia) now showing unanimous support. 


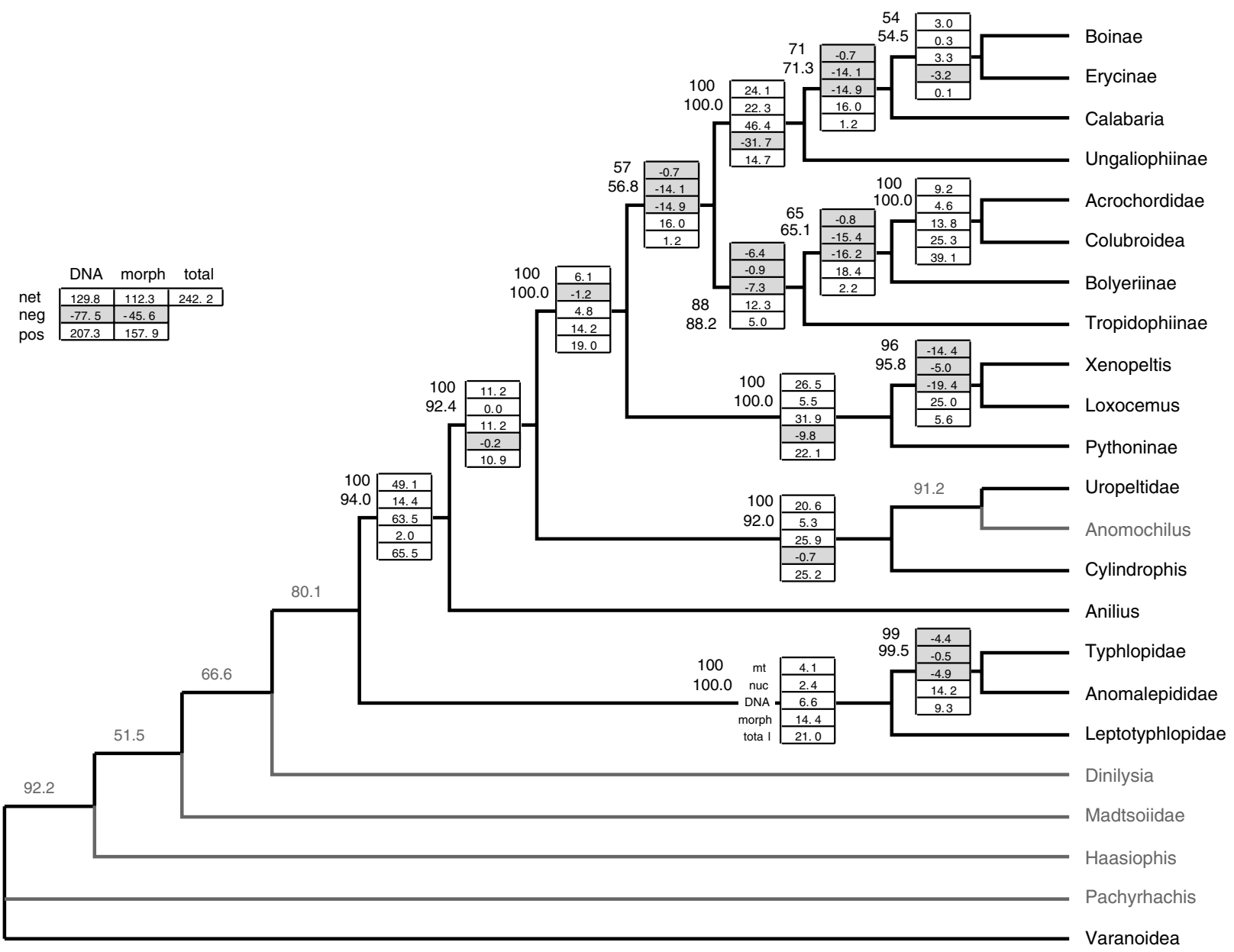

Figure 6 ML tree $(-\operatorname{lnL}=30464.989)$ from the Mkv + HKYg analysis, employing the 'Markovian' model for morphology and separate HKYg models for mitochondrial and nuclear data sets. The likelihood weighted majority rule consensus support for each clade in the complete (23-taxon) tree is shown in italics. This weighted consensus had the same topology as the ML tree. The support and PLS values for each clade in the subtree including only the 18 'extant' taxa are shown in bold. The five morphology-only taxa are in grey. Box at left shows totals split into positive and negative values for DNA and morphology.

The ML analysis employing the NCMr morphology model and the HKYg molecular models (NCMr+HKYg) yielded a best tree (Fig. 7) with $-\mathrm{lnL}=28571.334$ (NCMr component $-\operatorname{lnL}=962.642$, DNA component $-\operatorname{lnL}=27608.693$ ). This exhibited a few more differences from the parsimony tree. Again, tropidophiines appear higher up than in the parsimony tree; in this tree they emerged (weakly) as the sister group to bolyeriines alone. However, compared with the parsimony (and Mkv $+\mathrm{HKYg}$ ) tree, there was a change in the position of Loxocemus, which clustered with pythonines rather than Xenopeltis, as in recent molecular studies (Wilcox et al., 2002; Lawson et al., 2004; Vidal \& Hedges, 2004). There was also some minor rearrangement of the relative positions of advanced snakes, with tropidophiines and bolyeriines not forming a clade with caenophidians.

The Bayesian MCMC tree topology was very similar to that obtained in the Mkv + HKYg analysis, with only one different node (each alternative was poorly supported: posterior probability of 51\% versus likelihood weighting of 54\%); posterior probabilities are displayed on that tree (Fig. 8). Em- pirically the likelihood weights are quite well correlated with MCMC frequencies (posteriors) obtained below $(\mathrm{cc}=0.968)$, as would be expected given that both are a function of relative likelihoods (Strimmer \& Rambaut, 2002). The close match of our Mkv + HKYg model ML topology retrieved above with the consensus topology from MrBayes (using a matching model structure) and the close correlations between the likelihood weighted values and the posterior probabilities, suggest that the above manual procedure of $-\operatorname{lnL}$ calculation and topology searches were reasonably efficient and the pool of near-optimal trees reasonably complete.

The results for Scolecophidia are interesting because in the parsimony analysis, there was only morphological support for monophyly, with disagreement from both molecular partitions (see above). However, each scolecophidian taxon is at the end of a long branch, and the likelihood analysis (which should account for molecular evolution across long branches better than MP; Felsenstein, 2004) now reveals support for scolecophidian monophyly and thus improves congruence between all data partitions. The only strongly-supported difference to the 


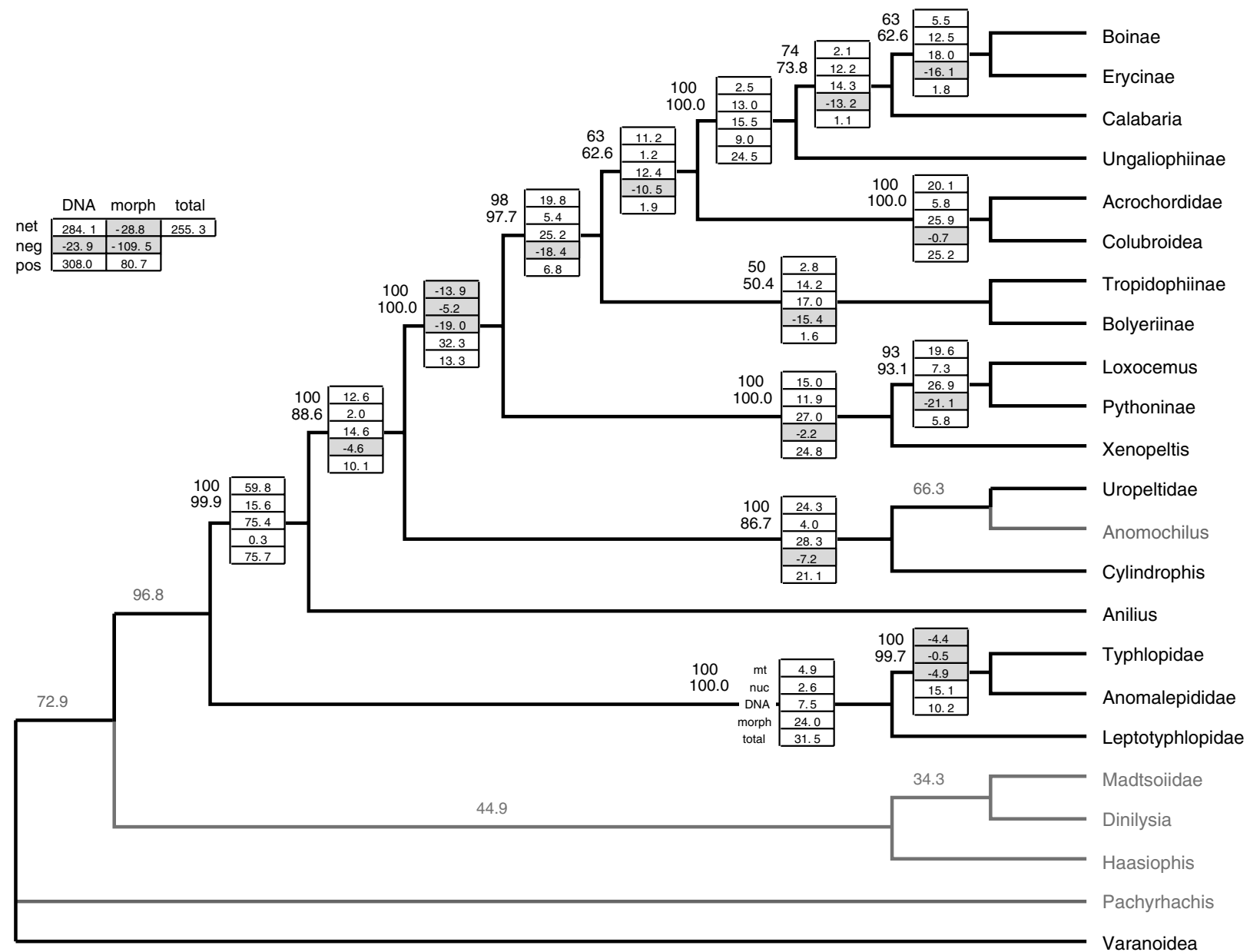

Figure $7 \mathrm{ML}$ tree $(-\operatorname{lnL}=28571.334)$ from the NCMr + HKYg analysis, employing the 'No Common Mechanism' model for morphology and separate HKYg models for mitochondrial and nuclear DNA. The likelihood weighted support for each clade in the complete (23-taxon) tree is shown in italics. This weighted consensus had the same topology as the ML tree. The five morphology-only taxa are in grey. The support and PLS values for each clade in the subtree including only the 18 'extant' taxa are shown in bold. The PLS boxes and box at left as for figure 5 .

MP analysis was the position of trophidophiines, which occupy a higher position within macrostomatans, close to caenophidians and bolyeriines.

Unweighted parsimony analysis treats all informative traits (morphological and molecular) equally, and larger data sets (e.g. long sequence data) will often swamp smaller data sets (e.g. morphology). The relative influence of morphological and molecular data sets in combined ML analyses has not yet been properly explored. The relatively basal position found for the tropidophiines in the MP analyses is largely consistent with analyses of molecular data alone, and suggests a strong influence of this data set. The higher position of tropidophiines found in both ML analyses is more consistent with the morphological signal alone, and suggests that both ML analyses accorded more weight to the morphological data (than did the parsimony analyses). Furthermore, PLS values and topological differences between the ML analyses indicated that the NCMr $+\mathrm{HKYg}$ analysis weighted morphology less than did the Mkv + HKYg analysis. The PLS values for morphology in the Mkv + HKYg analysis were larger in absolute value and more frequently positive, again suggesting that morpho- logy had a stronger influence in that analysis. In the nodes where the two ML analyses differ, the Mkv-HKYg analysis retrieved a topology more consistent with the morphological data (e.g. Xenopeltis-Loxocemus clade and a slightly higher position for tropidophiines) while the $\mathrm{NCMr}+\mathrm{HKYg}$ analysis retrieved a topology more consistent with the molecular data (e.g. Loxocemus-pythonine clade and a slightly lower position for tropidophiines). These observations suggested that the relative signal strength of the morphological and molecular data differs in MP and ML analyses, and even between different ML analyses.

\section{Discussion}

\section{Weighting morphology and molecules in parsimony and likelihood}

In order to evaluate the relative signal strength of morphology and molecules, 10000 random trees were generated, and the morphological and molecular data sets optimised onto these trees (calculated as described in methods). The distribution of 


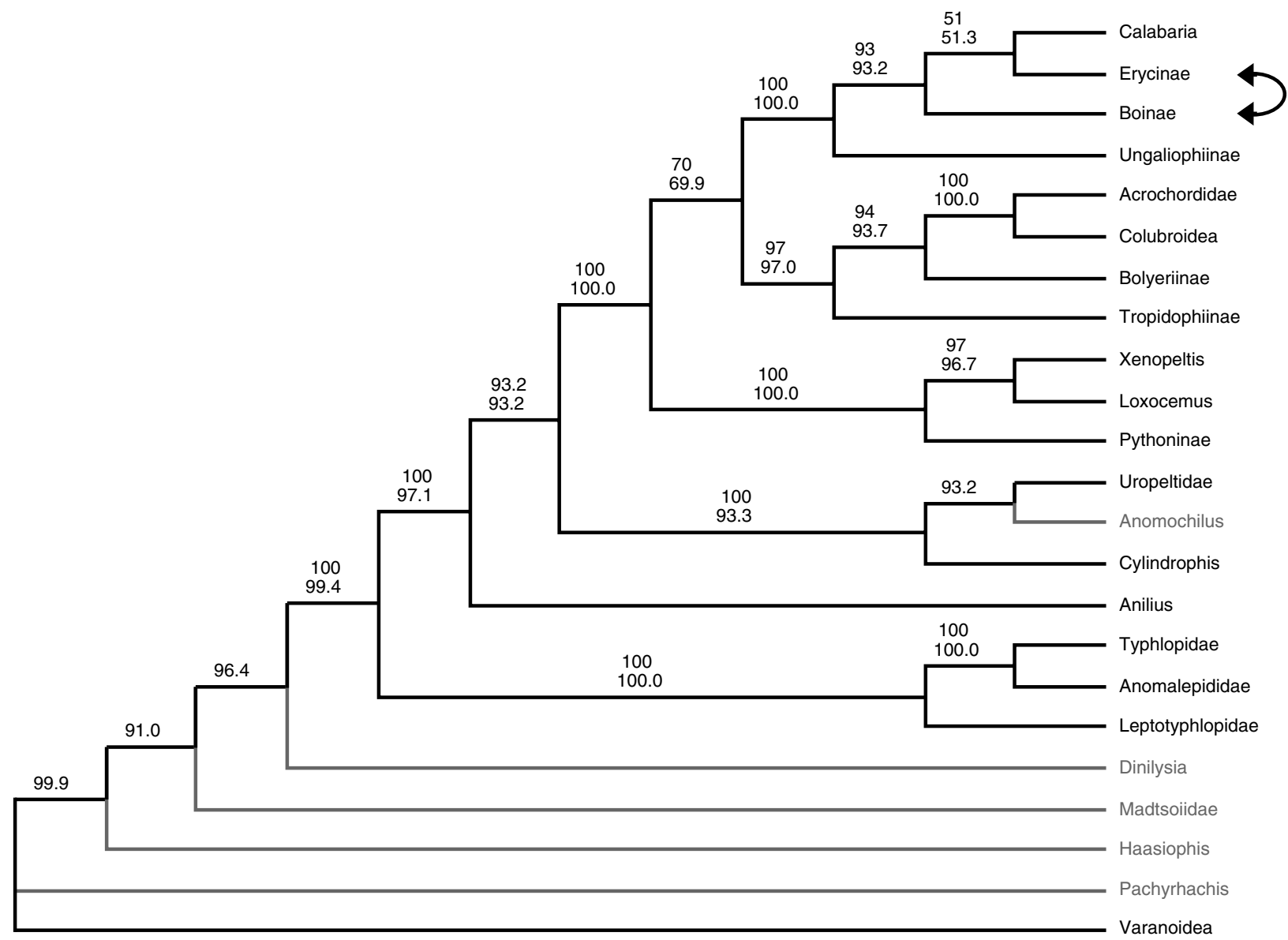

Figure 8 The majority-rule tree from the 95000 sampled trees from the MCMC analysis in MrBayes (Ronquist \& Huelsenbeck, 2003); note the similarity to Fig. 5. This analysis employed the standard Markovian model for morphology in MrBayes (a Mkv-type model) and separate HKYg models for mitochondrial and nuclear data sets. The posteriors for each clade in the full (23-taxon) data set are shown in normal font; posteriors for each clade in the reduced (18-taxon) data set are shown in bold. The five morphology-only taxa are in grey. Manual ML analysis (Fig. 4) differs by switching Boinae and Calabaria.

tree scores (lengths) under parsimony for the morphological and combined molecular partitions are plotted in Fig. 9a, with the mean score set to zero. Under unweighted parsimony, the morphological data has a narrower distribution of tree lengths than does the combined molecular data (ratio of standard deviations, $0.7: 1$ ), indicating a weaker phylogenetic signal in the morphological data. Here we use standard deviation as an index rather than an absolute measure of 'weight'. The morphological data will generally arbitrate between any two trees less strongly than will the molecular data. These results are consistent with the results of the parsimony analysis, which show a tree topology heavily influenced by the molecular data (suggested by the low position of tropidophiines). However, it should be noted that the morphological signal is still surprisingly strong given the relative numbers of parsimony-informative characters in the two data sets. Here, a morphological data set of 263 characters (260 parsimony-informative) has almost as much signal strength as a concatenated molecular data set of 4161 nucleotides (1263 parsimony-informative).

For the same 10000 random trees, the distribution of tree scores (likelihoods) under ML optimisation is shown in Fig 9b. The likelihood distributions for morphology (under the NCMr and Mkv models) and molecules are plotted separately with the mean likelihood of each set to zero. Under ML, there is an increase in the spread (i.e. signal strength) of the morphological data relative to the molecular data. The S.D. of the morphological data under the NCMr model is approximately equal to the S.D. of the molecular data (S.D. ratio 1.0), while the S.D. under the Mkv model is 1.3 times that of the molecular data. The standard deviations of the mitochondrial and nuclear partitions are also shown in Fig. 9c. Thus, compared to the parsimony analyses, in likelihood analyses, the morphological data were accorded the same (NCMr model), nearly one-and-a-half times (Mkv model) or more than twice (Mkv600 model) as much weight as DNA data. The trees found in both the ML analyses thus have topologies more influenced by the morphological data, as suggested by the higher position of tropidophiines. Also, the analysis employing the Mkv morphology model is the most consistent with the morphological tree, with an extremely high position for tropidophiines (near caenophidians) and a Xenopeltis-Loxocemus clade.

The above results demonstrate major differences in relative weighting of morphological versus molecular data in the unweighted (i.e. equally weighted) parsimony, $\mathrm{NCMr}+\mathrm{HKYg}$ 
A

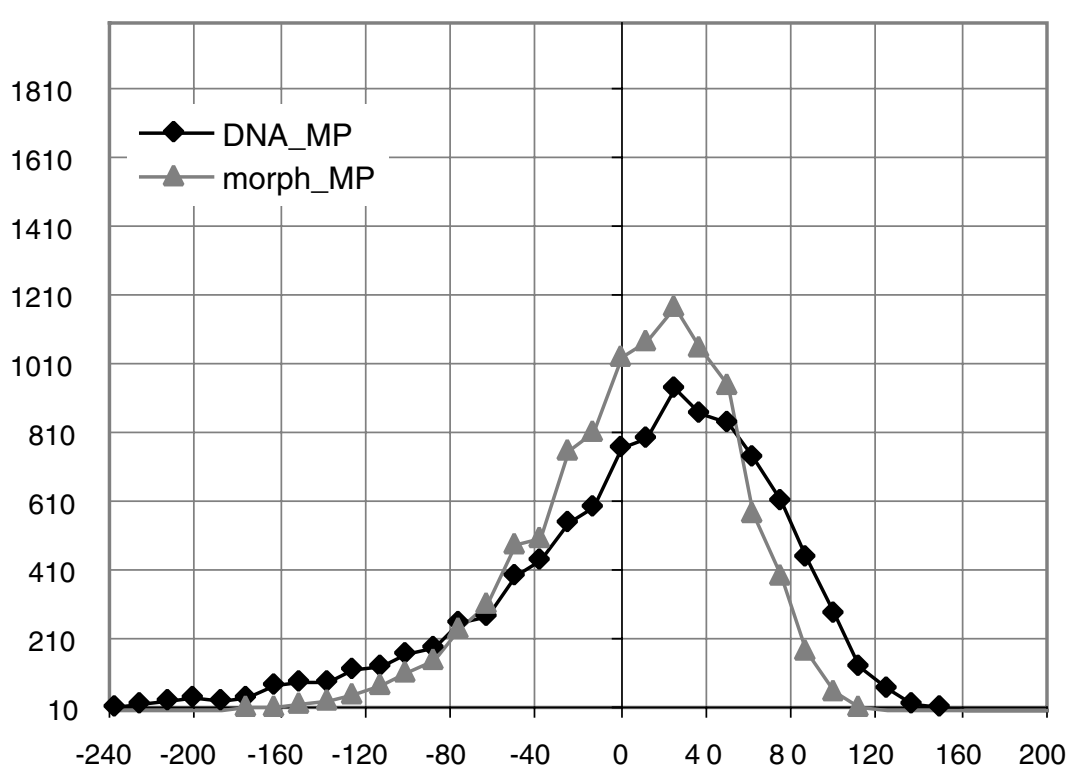

B

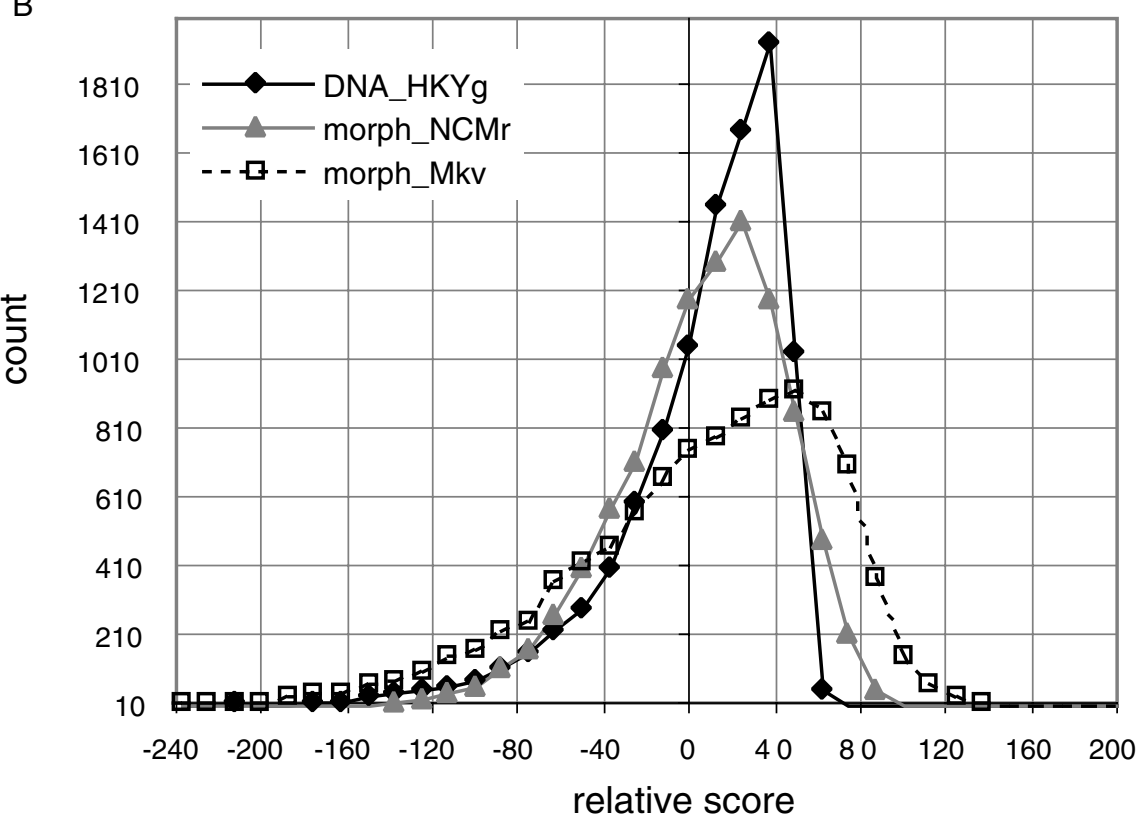

C

\begin{tabular}{ll|cccc}
\multicolumn{2}{r|}{ morphology } & & DNA & mtDNA & nuclear \\
model & s.d. & model & s.d. & s.d. & s.d. \\
\hline MP & 1.00 & MP & 1.44 & 1.25 & 0.24 \\
NCMr & 0.84 & HKYg & 0.85 & 0.66 & 0.24 \\
Mkv & 1.33 & HKYg & 0.85 & 0.66 & 0.24 \\
Mkv600 & 2.30 & & & &
\end{tabular}

Figure 9 (a) The relative signal strength of the morphological and molecular (combined mitochondrial and nuclear) partitions in parsimony analysis, as measured by the distribution of tree lengths for each data set for 10000 random trees. The curves have been superimposed by setting the mean tree length to zero. (b). The relative signal strength of the morphological and molecular (combined mitochondrial and nuclear) partitions in ML analyses, as measured by the distribution of tree scores (-InLs) for each data set for 10 ooo random trees. The curves have been superimposed by setting the mean tree score to zero. The two curves for the morphological data denote the results of the NCMr and Mkv models; the molecular data was optimised via the HKYg model. (c) The standard deviations for the morphological and molecular data sets, relative to morphology $M P=1$. The two components of the molecular data set graphed in (a) and (b) are also shown. Total DNA is sum of separate HKYg model scores for mtDNA and nuclear partitions. 


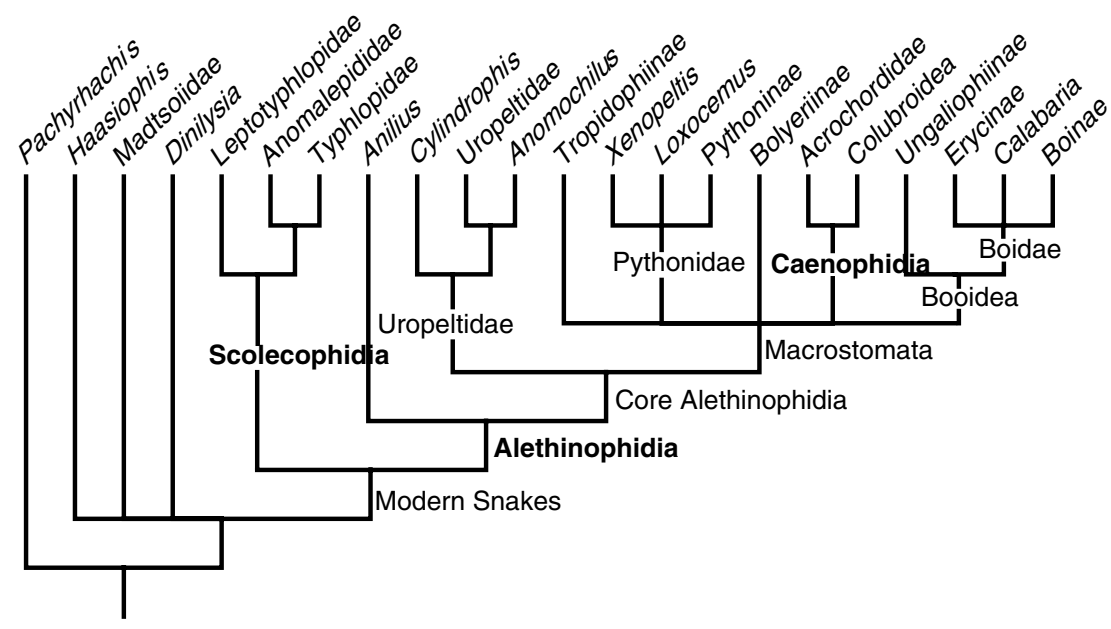

Figure 10 A strict consensus of the MP, $\mathrm{NCMr}+\mathrm{HKYg}$, and Mkv + HKYg trees, showing the apparent robustness (insensitivity) of the data set to differences in character weighting and models. Clades in bold are especially robust and are given phylogenetic definitions in the main text.

and Mkv $+\mathrm{HKYg}$ analyses. These differences result in predictable changes in tree topologies and the distribution of support across data sets. As the relative signal strength of the morpho$\operatorname{logy}$ is upweighted, this data set begins to drive tree topology more strongly. The partitioned support values for morphology accordingly become both relatively larger in absolute value, and more frequently positive; those for the molecular data sets become more frequently negative (Figs 4-7). This highlights the problem of character weighting, especially in ML analyses. Given the plethora of options for differential weighting (both between and within morphological and molecular data sets) equal weighting of each character is often seen as invoking the fewest assumptions (e.g. Kluge, 1998). However, strict equal weighting is only possible if the parsimony (or the parsimonylike NCMr model) is applied to all characters; only then can morphological and molecular characters be considered equivalent currency because the same model is applied to both.

In combined ML analyses, different likelihood models must be applied to morphological and molecular characters; this means a morphological character cannot be equated to a molecular character, and makes strict equal weighting (if desired) an impossible aim. When there is extensive conflict between morphological and molecular data, tree topology and support can be greatly influenced by different weighting schemes for these data partitions. The different models for morphology accord different relative weight to these characters, and there is no obvious criterion to decide which resultant weighting scheme is most appropriate and thus, which resultant trees are to be preferred. Effectively $r$ controls the character weight in the NCMr model; whereas the situation is more complex for Mkv models. Of the two ML models for morphology investigated here, NCMr is closer than is Mkv to unweighted parsimony not only in choice of trees (Fig. 2), but also in the relative weight assigned to morphological characters. We have also made no formal attempt to test the fit of morphological data to these two models (see caveats in 'Likelihood Ana- lyses'). It is not clear how this could be done or interpreted (Steel \& Penny, 2000), therefore the '. . proper statistical procedures ...' discussed in Edwards (1992; see also Goldman, 1990) are somewhat moot here. Given the problems in justifying model choice in the first place, favouring non-extreme weighting models could be a useful criterion to consider.

While there is considerable conflict between morphological and the molecular partitions (either separately or combined), there is considerable congruence between the two molecular partitions, especially in the likelihood analyses. In the Mkv + HKYg analysis, for instance, the mitochondrial and nuclear PLS values have the same sign for 14/15 nodes, whereas the combined DNA and the morphology have the same sign in only $4 / 15$ nodes. The corresponding figures for the $\mathrm{NCMr}+\mathrm{HKYg}$ are $15 / 15$ and $3 / 15$. However, in the MP analysis, corresponding figures are 6/15 and 6/15; the decreased congruence between molecular partitions in MP analyses suggests that this method is less appropriate for molecular data. In random trees (see Fig. 2), rank correlation between morphology and mtDNA or nuclear DNA partitions ranges from 0.318 to 0.400 (depending on morphology model) but is 0.666 between the molecular partitions. Such high levels of conflict can create complex, unstable tree support 'landscapes' with multiple local optima so that minor differences in models and data weighting can cause flips between very different preferred topologies.

Despite this prognosis, the empirical results here are encouraging. There is strong conflict between the morphological and molecular data plus a twofold difference in relative weighting between these data sets across the MP and ML analyses, yet the topology of most of the tree remained consistent. All analyses were much more resolved than the taxonomic congruence tree (Fig. 3d) and retain the monophyly of the following groups (Fig. 10): modern snakes, scolecophidians, typhlopids + anomalepidids, alethinophidians, core alethinophidians, uropeltids (sensu McDowell, 1975: Cylindrophis, Anomochilus, uropeltines), macrostomatans (sensu 
lato, e.g. Rieppel, 1988; Lee \& Scanlon, 2002), booids, boids, pythonids (sensu lato including Loxocemus and Xenopeltis) and caenophidians. The apparent conflict between the data sets regarding scolecophidian monophyly in the parsimony analyses disappears in the ML analyses, suggesting that the model-based methods are causing the molecular data to converge on the well-supported morphological tree. The novel position of the uropeltine-cyllindrophiine clade (= Uropeltidae s.1.) is a stable outcome of all MP and ML combined analyses here. Uropeltidae always emerges as the second-most basal clade of Alethinophidians, above Anilius. Morphological data alone place the Uropeltidae with Anilius, as basal alethinophidians, while molecular data alone, places this clade in a poorly resolved polytomy with all macrostomatans apart from tropidophiines (Fig. 1). Examination of PBS/PLS values for the relevant clades in the combined analyses shows the influence of both data sets: the morphological data set is responsible for Uropeltidae 'down' to a relatively basal position outside of macrostomatans, whereas the molecular data set is responsible for pulling Uropeltidae 'up' away from Anilius. The result is that in all analyses Uropeltidae emerges in a stable position consistent with (or at least not strongly contradicted by) each data set. In contrast, the position of tropidophiines is particularly labile in the different combined analyses, either closely reflecting the morphological or molecular results, and suggests that there is no novel position for tropidophiines consistent with both data sets.

\section{Snake phylogeny and taxonomy}

By highlighting areas of congruence and, equally importantly, incongruence, the above results have important implications for the higher-level phylogeny and classification of snakes. The strongly divergent signals of the molecular and morphological data mean that few clades are unanimously supported; it is these few which are discussed and formally defined below. Alethinophidia has positive support from all three data sets in all analyses (parsimony, $\mathrm{NCMr}+\mathrm{HKYg}$ and $\mathrm{Mkv}+\mathrm{HKYg}$ ), and can be considered to be extremely well-corroborated. Similarly, Caenophidia has positive support from all three data sets in the parsimony and Mkv + HKYg analysis, and is not strongly contradicted by any data set in the $\mathrm{NCMr}+\mathrm{HKYg}$ analysis. Scolecophidia has support from all data sets in both ML analyses and can also be considered robust; as expected it is also supported by analyses of the morphological data alone (e.g. Lee \& Scanlon, 2002), and likelihood analyses of the combined molecular data set alone (results not shown). The negative molecular PBS values for Scolecophidia in the parsimony analysis is probably an artefact of parsimony's inability to deal adequately with long terminal branches (Felsenstein, 2004). All other clades are strongly contradicted by at least one data set in at least two of the three analyses, and as such must be seen as provisional. With Scolecophidia, Alethinophidia, and Caenophidia all robustly supported by the three data sets, the major remaining uncertainties in snake phylogeny concern the interrelationships of basal alethinophidians (anilioids and booids). Such reservations are expected, given that the major conflicts between morphological and molecular data have involved this part of the tree, especially the position of trop- idophiines, ungaliophiines, Xenopeltis and Loxocemus (see Introduction).

We here provide phylogenetic definitions (Cantino \& de Queiroz, 2000) for the three clades most robustly retrieved in this study (Fig. 10); these are well-corroborated in all three analyses and are supported by all data sets in the ML analyses. The definitions are designed to be stable (in terms of included taxa) in the face of remaining uncertainties in snake phylogeny, especially regarding basal relationships of alethinophidians (this study), and basal relationships within caenophidians (e.g. Kraus \& Brown, 1996; Kelly et al., 2003). However, the definitions have 'designated phylogenetic contexts' (Bryant, 1997; Lee, 1998) and are inapplicable (nonsensical) if the current concepts of Scolecophida, Alethinophidia and Caenophidia are refuted.

Scolecophidia. Various names were proposed in the 19th and early 20th centuries for essentially this group, comprising the small-mouthed, fossorial 'blind snakes', 'worm snakes' and 'thread snakes'. The most widely used term (Scolecophidia) was coined as an informal name by Duméril and Bibron (1844), later adopted formally by Cope (1864), and has been used for the past half-century with almost no variation in content (Lee \& Scanlon, 2002). The name is here defined as a node-based entity, the most recent common ancestor of Leptotyphlops albifrons, Anomalepis mexicanus, Typhlops lumbricalis, to the exclusion of Anilius scytale, Uropeltis ceylanica, Tropidophis melanurus and Coluber constrictor. These species are all the type species of their eponymous Linnaean 'families' (McDiarmid et al., 1999). Morphological diagnosis: see Lee and Scanlon (2002).

Alethinophidia. This term was first used by Nopcsa (1923) to comprise all modern snakes other than scolecophidians (which Nopcsa termed angiostomatans); both of these extant branches were proposed to be derived from an implicitly paraphyletic assemblage of extinct basal snakes (Cholophidia; Nopcsa, 1923). Alethinophidia is here defined as a node-based entity, the most recent common ancestor of Anilius scytale, Uropeltis ceylanicus, Tropidophis melanurus, and Coluber constrictor, to the exclusion of Leptotyphlops albifrons, Anomalepis mexicanus, Typhlops lumbricalis. These species are all the types of their eponymous Linnaean 'families' (McDiarmid et al., 1999). Morphological diagnosis: see Lee and Scanlon (2002).

Caenophidia. This term was first used as part of Hoffstetter's (1939) grade-based subdivision of Alethinophidia whereby primitive members were termed henophidians, and forms seen as more 'specialised' or 'advanced' (e.g. see Nopcsa, 1923) were termed caenophidians. As with many 'horizontal' divisions, the primitive grade was explicitly paraphyletic and not now formally recognised, but the advanced grade coincides with a clade. Caenophidia is here defined as a node-based entity, the most recent common ancestor of $\mathrm{Ac}$ rochordus javanicus, Vipera aspis and Coluber constrictor to the exclusion of Bolyeria multocarinata, Boa constrictor, Python molurus and Uropeltis ceylanicus. These species are all the types of their eponymous Linnaean 'families' (McDiarmid et al., 1999). Morphological diagnosis: see Lee and Scanlon (2002). 


\section{Acknowledgements}

Portions of this work were presented at a Linnean Society symposium in honour of Garth Underwood (April 2004), and ML would like to acknowledge Garth Underwood's encouragement, inspiration and immense personal contributions in this area, as well as Roger Thorpe and the conference organisers for making attendance possible. We thank Tod Reeder for allowing us to use and deposit onto Genbank his unpublished rDNA sequences of Varanus, Van Wallach for discussion of snake taxonomy, Christopher Kelly and (especially) an anonymous referee for extensive comments, and the Australian Research Council for funding.

\section{References}

BAKER, R.H. \& DESALLE, R. 1997. Multiple sources of character information and the phylogeny of Hawaiian drosophilids. Systematic Biology 46, 654-673.

BARKER, F.K. \& LUTZONI, F.M. 2002. The utility of the incongruence length difference test. Systematic Biology 51, 625-657.

Barrett, M., Donoghue, M.J. \& Sober, E. 1991. Against consensus. Systematic Biology 40, 486-493.

Bellairs, A.D'A. \& Underwood, G. 1951. The origin of snakes. Biological Reviews 26, 193-237.

BREMER, K. 1988. The limits of amino acid sequence data in angiosperm phylogenetic reconstruction. Evolution 42, 795-803.

BRomham, L., WoOlFit, M., LeE, M.S.Y. \& Rambaut, A. 2002. Testing the relationship between morphological and molecular rates of change among phylogenies. Evolution 56, 1921-1930.

BRYANT, H.N. 1997. Cladistic information in phylogenetic definitions and designated phylogenetic contexts for the use of taxon names. Biological Journal of the Linnean Society 62, 497-503.

Bull, J.J., Huelsenbeck, J.P., Cunningham, C.W., Swofford, D.L. \& WADDELL, P.J. 1993. Partitioning and combining data in phylogenetic analysis. Systematic Biology 42, 384-397.

CALDWELL, M.W. 1999. Squamate phylogeny and the relationships of snakes and mosasauroids. Zoological Journal of the Linnean Society 125, 115-147.

CAMP, C.L. 1923. Classification of the lizards. Bulletin of the American Museum of Natural History 48, 289-481.

Cantino, P.D. \& De QueIroz, K. 2000. Phylocode: A Phylogenetic Code of Biological Nomenclature. http://www.ohiou.edu/ phylocode.

COPE, E.D. 1864. On the characters of the higher groups of Reptilia Squamata - and especially of the Diploglossa. Proceedings of the Academy of Natural Sciences, Philadelphia 1864, 224-231.

CundALl, D. \& GREENE, H.W. 1982. Evolution of the feeding apparatus in alethinophidian snakes. American Zoologist 22, 924.

Cundall, D., \& Greene, H.W. 2000. Feeding in snakes. In: SChWEnK, K., Ed., Feeding: Form, Function, and Evolution in Tetrapod Vertebrates. Academic Press, San Diego, pp. 293-333.

Cundall, D., Wallach, V. \& Rossman, D.A. 1993. The systematic relationships of the snake genus Anomochilus. Zoological Journal of the Linnean Society 109, 275-299.

Dowling, H.G. \& Duellman, W.E. 1978. Systematic Herpetology: A Synopsis of Families and Higher Categories. HISS Publications in Herpetology No. 7. HISS Publications, New York.

DUMÉRIL, A.-M.-C. \& BIBRON, G. 1844. Erpetologie générale ou histoire naturelle complète des reptiles. Vol. 6. Librarie Encyclopédique de Roret, Paris.

EDWARDS, A.F.W. 1992. Likelihood. Expanded edition. Johns Hopkins University Press.

FELSENSTEIN, J. 1981. Evolutionary trees from DNA sequences: a maximum likelihood approach. Journal of Molecular Evolution 17, 368-376.

FELSENSTEIN, J. 1992. Phylogenies from restriction sites: a maximumlikelihood approach. Evolution 46, 159-173.
FELSEnSteIn, J. 2004. Inferring phylogenies. Sinauer, Sunderland, MA.

GATESY, J., O'GRADY, P. \& BAKER, R.H. 1999. Corroboration among data sets in simultaneous analysis: hidden support for phylogenetic relationships among higher-level artiodactyl taxa. Cladistics $\mathbf{1 5}$, 271-313.

Gatesy, J., Amato, G., Norell, M., DeSalle, R. \& Hayashi, C. 2003. Combined support for wholesale taxonomic atavism in gavialine crocodylians. Systematic Biology 52, 403-422.

GoldMAN, N. 1990. Maximum likelihood inference of phylogenetic trees, with special reference to a poisson process model of DNA substitution and to parsimony analyses. Systematic Biology 39, $345-361$.

GREENE, H.W. 1983. Dietary correlates of the origin and radiation of snakes. American Zoologist 23, 431-441.

GreENE, H.W. 1997. Snakes - The Evolution of Mystery in Nature. University of California Press, Berkeley.

HaSegawa, M., Kishino, H. \& YANO, T. 1985. Dating the human-ape splitting by a molecular clock of mitochondrial DNA. Journal of Molecular Evolution 22, 160-174.

HeISE, P.J., MAXson, L.R., Dowling, H.G. \& Hedges, S.B. 1995. Higher-level snake phylogeny inferred from mitochondrial DNA sequences of 12S rRNA and 16S rRNA genes. Molecular Biology and Evolution 12, 259-265.

HILLIS, D.M. 1996. Inferring complex phylogenies. Nature 383, 130-131.

HOFFSTETTER, R. 1939. Contribution a l'étude des Elapidae actuels et fossiles et de l'ostéologie des ophidiens. Archives du Museum D'Histoire Naturelle de Lyon 15, 1-78.

HuELSENBECK, J.P. \& RonQuist, F.R. 2001. MRBAYES: Bayesian inference of phylogenetic trees. Bioinformatics 17, 754-755.

Jamieson, B.G.M., Tillier, S., Tillier, A., Justine, J.-L., Ling, E., James, S., McDonald, K. \& Hugall, A.F. 2002. Phylogeny of the Megascolecidae and Crassiclitellata (Oligochaeta, Annelida): Combined versus partitioned analysis using nuclear (28S) and mitochondrial (12S, 16S) rDNA. Zoosystema 24, 707734.

Jerminn, L.S., Olsen, G., Mengersen, K.L. \& Easteal, S. 1997. Majority-rule consensus of phylogenetic trees obtained by maximum-likelihood analysis.Molecular Biology and Evolution 14, 1296-1302.

JUKES, T.H. \& CANTOR, C.R. 1969. Evolution of protein molecules. In: MunRo, H.N., Ed., Mammalian Protein Metabolism. Academic Press, New York, pp. 21-132.

Kelly, C.M., BarKeR, N.P. \& VILlet, M.H. 2003. Phylogenetics of advanced snakes (Caenophidia) based on four mitochondrial genes. Systematic Biology 52, 439-459.

KLUGE, A.G. 1989. A concern for evidence and a phylogenetic hypothesis of relationships among Epicrates (Boidae, Serpentes). Systematic Zoology 38, 7-25.

KLUGE, A.G. 1991. Boine snake phylogeny and research cycles. Miscellaneous Publications of the Museum of Zoology, University of Michigan 178, 1-58.

KLUGE, A.G. 1993. Calabaria and the phylogeny of erycine snakes. Zoological Journal of the Linnean Society 107, 293-351.

KLUGE, A.G. 1998. Sophisticated falsificationism and research cycles: consequences for differential character weighting in phylogenetic systematics. Zoologica Scripta 26, 349-360.

KraUS, F. \& Brown, W.M. 1998. Phylogenetic relationships of colubroid snakes based on mitochondrial DNA sequences. Zoological Journal of the Linnean Society 122, 455-487.

LaWson, R., SLOWINSKI, J.B. \& BurbrinK, F.T. 2004. A molecular approach to discerning the phylogenetic placement of the enigmatic snake Xenophidion schaeferi among the Alethinophidia. Journal of Zoology, London 263, 1-10.

LEE, M.S.Y. 1997. The phylogeny of varanoid lizards and the affinities of snakes. Philosphical Transactions of the Royal Society of London, Series B 352, 53-91. 
LEE, M.S.Y. 1998. Convergent evolution and character correlation in burrowing reptiles: towards a resolution of squamate phylogeny. Biological Journal of the Linnean Society 65, 369-453.

LEE, M.S.Y. 2005a. Squamate phylogeny revisited: taxon sampling, character analysis, and data congruence. Organisms, Diversity and Evolution 5, 25-45.

LEE, M.S.Y. 2005b. Molecular evidence and snake origins. Biology Letters 1, 227-230.

LEE, M.S.Y. \& CALDWELL, M.W. 2000. Adriosaurus and the affinities of mosasaurs, dolichosaurs, and snakes. Journal of Paleontology 74, 915-937.

LEE, M.S.Y. \& HugaLL, A.F. 2003. Partitioned likelihood support and the evaluation of dataset conflict. Systematic Biology 52, 15-22.

LEE, M.S.Y. \& SCANLON, J.D. 2002. Snake phylogeny based on osteology, soft anatomy, and behaviour. Biological Reviews 77, 333 401.

LEWIS, P.O. 2001. A likelihood approach to estimating phylogeny from discrete morphological character data. Systematic Biology 50, 913-925.

McDiarmid, R.W., CAmpbell, J.A. \& Touré, T.A. 1999. Snake Species of the World: A Taxonomic and Geographic Reference. Volume 1. The Herpetologist's League, Washington, DC.

MCDOWELL, S.B. 1975. A catalogue of the snakes of New Guinea and the Solomons, with special reference to those in the Bernice P. Bishop Museum. Part II. Anilioidea and Pythoninae. Journal of Herpetology 9, 1-80.

MCDowell, S.B. 1987. Systematics. In: SeIGEL, R.A., Collins, J.T.C. \& NovaK, S.S., Eds., Snakes: Ecology and Evolutionary Biology. MacMillan, New York, pp. 1-50.

NIXON, K.C. \& CARPENTER, J.M. 1996. On simultaneous analysis. Cladistics 12, 221-241.

NoPCSA, F. 1923. Eidolosaurus und Pachyophis. Zwei neue NeocomReptilien. Palaeontographica 65, 99-154.

PosADA, D. \& CRANDALL, K. 1998. Modeltest: testing the model of DNA substitution. Bioinformatics 14, 817-818.

RAGE, J.-C. \& EsCuILliÉ, F. 2002. Eupodophis, new name for the genus Podophis Rage and Escuillié, 2000, an extinct bipedal snake, preoccupied by Podophis Wiegmann, 1834 (Lacertilia, Scincidae). Amphibia-Reptilia 23, 232-233.

Rage, J.-C. \& Escuillié, F. 2003. The Cenomanian: stage of hindlimbed snakes. Carnets de Géologie/Notebooks on Geology, Maintenon, Article 2003/01 (Reference number: CG2003_A01_JCR-FE). 11 pp. http://paleopolis.rediris.es/cg/ukindex.html

RIEPPEL, O. 1988. A review of the origin of snakes. Evolutionary Biology 22, 37-130.

RiePPel, O., ZAHER, H., TCHERnOV, E. \& POLCYN, M.J. 2003. The anatomy and relationships of Haasiophis terrasanctus, a fossil snake with well-developed hind limbs from the mid-Cretaceous of the Middle East. Journal of Paleontology 77, 536-558.

RonQuist, F. \& HuELSENBECK, J.P. 2003. MrBayes 3: Bayesian Phylogenetic Inference Under Mixed Models. Computer program and documentation. www.morphbank.ebc.uu.se/mrbayes.

SAVITZKY, A.H. 1983. Coadapted character complexes among snakes: fossoriality, piscivory, and durophagy. American Zoologist 23, 397-409.

SCANLON, J.D. 1996. Studies in the palaeontology and systematics of Australian snakes. Ph.D. dissertation, University of New South Wales, Sydney.

SCOTLAND, R.W., OlmSteAD, R.G. \& BenNeT, J.R. 2003. Phylogeny reconstruction: The role of morphology. Systematic Biology 52, $539-548$

SCHWENK, K. 1988. Comparative morphology of the lepidosaur tongue and its relevance to squamate phylogeny. In: ESTES, R. and PREGILl, G., Eds., Phylogenetic Relationships of the Lizard Families: Essays Commemorating Charles L. Camp. Stanford Univ. Press, Stanford, California, pp. 569-598.
SEO, T-K., Kishino, H. \& THORNE, J.L. 2005. Incorporating genespecific variation when inferring and evaluating optimal evolutionary tree topologies from multilocus sequence data. Proc. Natl. Acad. Sci. USA 102, 4436-4441.

SLOWINSKI, J.B. \& LAWSON, R. 2002. Snake phylogeny based on mitochondrial and nuclear genes. Molecular Phylogenetics and Evolution 24, 194-202.

SORENSON, M. 1999. TreeRot Version 2. Computer program and documentation. Distributed by the author, Boston University.

Steel, M. \& PenNy, D. 2000. Parsimony, likelihood, and the role of models in molecular phylogenetics. Molecular Biology and Evolution 17, 839-850.

STRIMMER, K. \& RAMBAUT, A. 2002. Inferring confidence sets of possibly misspecified gene trees. Proceedings of the Royal Society of London, Series B 269, 137-142.

SWOFFORD, D.L. 2002. PAUP* - Phylogenetic Analysis Using Parsimony ( ${ }^{*}$ and other methods). Version 4 , beta versions $8-10$. Sinauer, Sunderland MA.

TCHERNOV, E., RIEPPEL, O., ZAHER, H., POLCYN, M.J. \& JACOBS, L.L. 2000. A fossil snake with limbs. Science 287, 2010-2012.

THOMAS, R.A. 1989. The relationships of Antillean Typhlops (Serpentes: Typhlopidae) and the description of three new Hispaniolan species. In: Woods, C.A., Ed., Biogeography of the West Indies: Past, Present, and Future. Sandhill Crane Press, Gainesville, Florida, pp. 409-432.

THOMPSON, J., GiBson, T. \& HigGINs, D. 1997. Clustal Wversion 1.7. Program and documentation. www.es.embnet.org/Doc/clustalw/

Townsend, T.M., LARSON, A., LouIs, E. \& MaCEY, R.J. 2004. Molecular phylogenetics of Squamata: the position of snakes, Amphisbaenians, and Dibamids, and the root of the squamate tree. Systematic Biology 53, 735-757.

TUFFLEY, C. \& STEEL, M. 1997. Links between maximum likelihood and maximum parsimony under a simple model of site substitution. Bulletin of Mathemtical Biology 59, 581-607.

UNDERWOOD, G. 1967. A Contribution to the Classification of Snakes. Publication No. 653. British Museum (Natural History), London.

VIDAL, N. \& HEDGES, S.B. 2002. Higher-level relationships of snakes inferred from four nuclear and mitochondrial genes. Comptes Rendes, Biologies 325, 977-985.

VIDAL, N. \& HEDGES, S.B. 2004. Molecular evidence for a terrestrial origin of snakes. Biology Letters 271, 226-229.

WAhlberg, N., Braby, M.F., Brower, A.V.Z., De Jong, R., LeE, M.-M., Nylin, S., Pierce, N., Sperling, F.A.H., Vilda, R., WARREN, A.D. \& ZAKHAROV, E. 2005. Synergistic effects of combining morphological and molecular data in resolving the phylogeny of butterflies and skippers. Proceedings: Biological Sciences 272, 1577-1586.

WALLACH, V. 1998. The visceral anatomy of blindsnakes and wormsnakes and its systematic implications (Serpentes: Anomalepididae, Typhlopidae, Leptotyphlopidae). Ph.D. dissertation, Northeastern University, Boston, MA.

WALLS, G.L. 1940. Ophthalmological implications for the early history of snakes. Copeia 1940, 1-8.

WilcoX, T.P., ZWICKL, D.J., HEATH, T.A. \& HILlis, D.M. 2002. Phylogenetic relationships of the dwarf boas and a comparison of Bayesian and bootstrap measures of phylogenetic support. Molecular Phylogenetics and Evolution 25, 361-371.

YEATES, D. 1992. Why remove autapomorphies? Cladistics 8, 387389.

ZAHER, H. 1994. Les Tropidopheoidea (Serpentes: Alethinophidia) sont-ils réellement monophyletique? Arguments en faveur de leur polyphyletisme. Comptes Rendus de l'Académie des Sciences, Sciences de la Vie 317, 471-478.

ZAHER, H. \& RIEPPEL, O. 1999. The phylogenetic relationships of Pachyrhachis problematicus, and the evolution of limblessness in snakes (Lepidosauria, Squamata). Comptes Rendus de l'Académie des Sciences, Sciences de la terre et des planètes 329, 831-837. 